\title{
1 Pervasive conditional selection of driver mutations and modular epistasis networks in cancer
}

2 Jaime Iranzo ${ }^{1,2 *}$, George Gruenhagen ${ }^{3}$, Jorge Calle-Espinosa ${ }^{1}$, Eugene V. Koonin ${ }^{4 *}$

$3 \quad{ }^{1}$ Centro de Biotecnología y Genómica de Plantas, Universidad Politécnica de Madrid (UPM) - Instituto

4 Nacional de Investigación y Tecnología Agraria y Alimentaria (INIA), Madrid, Spain.

5 '2 Institute for Biocomputation and Physics of Complex Systems (BIFI), University of Zaragoza, 6 Zaragoza, Spain.

$7{ }^{3}$ Institute of Bioengineering and Biosciences, School of Biological Sciences, Georgia Institute of 8 Technology, Atlanta GA, USA.

$9{ }^{4}$ National Center for Biotechnology Information, National Library of Medicine, National Institutes of 10 Health, Bethesda MD, USA.

11 *Corresponding authors: koonin@ncbi.nlm.nih.gov, jaime.iranzo@upm.es

\section{Summary}

14 Cancer driver mutations often display mutual exclusion or co-occurrence, underscoring the key role of epistasis in carcinogenesis. However, estimating the magnitude of epistatic interactions and their quantitative effect on tumor evolution remains a challenge. We developed a method to quantify COnditional SELection on the Excess of Nonsynonymous Substitutions (Coselens) in cancer genes. Coselens infers the number of drivers per gene in different partitions of a cancer genomics dataset using covariance-based mutation models and determines whether coding mutations in a gene affect selection for drivers in any other gene. Using Coselens, we identified 296 conditionally selected gene pairs across 16 cancer types in the TCGA dataset. Conditional selection accounts for $25-50 \%$ of driver substitutions in tumors with $>2$ drivers. Conditionally co-selected genes form modular networks, whose structures challenge the traditional interpretation of within-pathway mutual exclusivity and across-pathway synergy, suggesting a more complex scenario, where gene-specific across-pathway interactions shape differentiated cancer subtypes.

Keywords: cancer genomics, conditional selection, epistasis, mutational landscape, cancer subtypes, 


\section{Introduction}

Cancer is a result of somatic evolution, during which cells accumulate driver mutations (drivers for short) that enable them to proliferate, bypassing cell cycle checkpoints and avoiding senescence, invade the surrounding tissues, escape the immune system, and ultimately disseminate to distant body locations(Hanahan and Weinberg, 2000; Stratton et al., 2009). By virtue of increasing the growth and survival rates of (pre)cancer cells, drivers are positively selected and can reach high prevalence within the tumor although not necessarily fixed (Garraway and Lander, 2013; Greaves and Maley, 2012; Nik-Zainal et al., 2012; Vogelstein et al., 2013; Williams et al., 2018). Concomitantly, (pre)cancer cells acquire passenger mutations which, despite not contributing to cancer initiation or progression, can spread by hitchhiking on drivers and typically are the dominant component of cancer mutational landscapes(Martincorena et al., 2017; Weghorn and Sunyaev, 2017).

The precise set of drivers acquired by a tumor determines some of its fundamental properties, such as metastatic potential and sensitivity to targeted therapies(Senft et al., 2017). Some of these properties can be related to the presence or absence of a single driver, whereas others result from the combination of multiple drivers that interact synergistically or interfere with each other, critically affecting tumor initiation, progression, and clinical outcome(Ashworth et al., 2011; Mina et al., 2017; Zhang et al., 2017). Two well-studied examples involve the synergy between activating mutations in oncogene RAS and inactivation of the tumor suppressor ATM, and the synthetic lethality of EGFR and $K R A S$ double mutants in lung and colon cancers. In the first case, inactivation of ATM allows tumor cells to overcome oncogene-induced senescence associated with RAS activation, and therefore exacerbates cell proliferation(Di Micco et al., 2006). In the second case, oncogenic mutations in KRAS make EGFR-mutant tumors resistant to therapies that target EGFR, but the synergistic deleterious effect of KRAS and EGFR mutations makes such escape clones inviable in the absence of the drug(Misale et al., 2014).

Epistatic interactions, such as those mentioned above, have the potential to substantially affect cancer somatic evolution by making selection on a driver conditional on the presence or absence of another driver (Wilkins et al., 2018). Epistasis results in patterns of co-occurrence when synergy between drivers enhances positive selection or mutual exclusivity when redundancy or antagonism diminish selection on the double mutant. Epistasis-driven conditional selection implies that past driver mutations affect the fitness of subsequent driver mutations and, with that, the trajectory of the tumor evolution.

Search for mutually exclusive mutations has been the focus of intense research in cancer genomics because such findings can lead to identification of synthetic lethal pairs and cancer-associated pathways, which are potential therapeutic targets(Babur et al., 2015; Jerby-Arnon et al., 2014; Kim et al., 2017; Leiserson et al., 2015; Matlak and Szczurek, 2017; Mina et al., 2017; Srihari et al., 2015; Wappett et al., 2016). However, because co-occurrence and mutual exclusivity can also result from systematic biases in tumor mutational load, their presence cannot be taken as unequivocal evidence of epistasis(van de Haar et al., 2019). Furthermore, the statistical frameworks commonly used to test for mutation co-occurrence and mutual exclusivity fail to provide quantitative estimates of biologically meaningful effect sizes, such as the extent to which epistasis modifies the fitness of a mutant clone and the subsequent probability that a mutation becomes a driver(Cannataro et al., 2018). 
Obtaining quantitative estimates of epistatic effects in a systematic and direct manner is extremely challenging although a recent study has approached this goal by conducting combinatorial RNAi screening in cell culture followed by image-based phenotyping of growth-related traits(Wang et al., 2014). An alternative approach is to quantify the contribution of epistasis to cancer development indirectly, through its effect on the conditional selection of driver mutations. This approach, which takes advantage of the recent surge in cancer genomic data, has the fundamental advantage of not relying on ad-hoc (in vitro) proxies for in vivo tumor fitness. A major difficulty, however, is that quantification of conditional selection requires disentangling the contributions of mutation and selection to cancer genomic landscapes, a challenging task that has only recently begun to be addressed by adapting tools from evolutionary genetics for cancer genomics(Cannataro et al., 2018; Martincorena et al., 2017; Persi et al., 2018; Weghorn and Sunyaev, 2017). Following this route, we developed a method that compares the mean number of drivers per tumor in a gene depending on whether a second gene harbors non-synonymous mutations, returning an estimate for the magnitude of conditional selection, while controlling for differences in mutational load and spectrum. Application of this method to somatic mutation data from more than 10,000 cancer exomes led to the identification of a complex network of cancer type-specific epistatic interactions, shedding light on the underlying genetic constraints that shape cancer development.

\section{Results and Discussion}

Detection of pairwise conditional selection among cancer genes

To investigate the role of conditional selection in cancer progression, we quantified how the number of driver substitutions (estimated as described below) in a cancer gene (the "query" gene) depended on the presence or absence of coding mutations in a second gene (the "split" gene). For each cancer type in The Cancer Genome Atlas (TCGA), we first identified the set of query genes by pooling all somatic mutations from the same cancer type and searching for genes for which the nonsynonymous-to-synonymous mutation ratio (dN/dS) indicated significant levels of positive selection (Supplementary Table 1). Query genes with coding mutations (nonsynonymous substitutions, truncations, and small indels) in $>25$ samples were additionally considered as split genes. Then, for all split-query gene pairs, we classified tumor samples into those that did and did not harbor coding mutations in the split gene and applied our evolutionary genetics-inspired method Coselens to estimate and compare the number of driver substitutions in the query gene in each of the groups (Figure 1a). In the first step, Coselens fits a mutational model to each group of samples and calculates the mean number of nonsynonymous and truncating substitutions that would be expected in the query gene in the absence of selection. Because purifying selection is extremely rare in non-hypermutated tumors, the number of positively selected (that is, driver) events can be readily obtained as the difference in the number of observed and expected mutations (see Methods for details). Then, Coselens implements a likelihood ratio test to assess whether the number of driver events significantly differs between samples that do and do not harbor coding mutations in the split gene.

Our analysis identified 296 gene pairs subject to significant conditional selection (FDR $\leq 5 \%$ ) in at least one cancer type (for the complete list, see Supplementary Table 2). Of these, 262 were deemed significant by the standard workflow and the rest were identified through restricted hypothesis 
testing of reciprocal interactions. The effect size distribution of significant pairs identified through restricted hypothesis testing closely resembles that of significant pairs detected through the more stringent, standard workflow (Figure 1b), suggesting that the former represent genuine cases of conditional selection. Overall, conditional selection affects 112 genes across 16 cancer types. In $70 \%$ of the conditionally selected gene pairs, coding mutations in the split gene increase the probability of finding driver mutations in the query gene; henceforth, we refer to these interactions as positive. In the remaining $30 \%$, coding mutations in the split gene decrease the probability to find driver mutations in the query gene, and henceforth, we refer to these interactions as negative (Figure 1c).

We further tested whether conditional selection in cancer gene pairs was reciprocal. Qualitatively, reciprocity implies that, if conditional selection is detected for a given split-query gene pair, it should be also detected for the reverse pair, in which the split and query genes are exchanged. Attending to this qualitative criterion, we found that $68 \%$ of the pairs that are subject to significant conditional selection are reciprocal (this fraction does not include reverse pairs that could not be tested due to insufficient number of coding mutations in one of the genes).

The magnitude of conditional selection varies among gene pairs, with coding mutations in the split gene typically associated with an increase or decrease of at least 0.15 driver substitutions per query gene per tumor (Figure 1b). In some extreme cases, conditional selection results in almost complete presence or absence of a driver mutation in the query gene. The pair of closely related oncogenes GNAQ and GNA11 is a good example of such extreme conditionality: driver substitutions in either GNAQ or GNA11 are found in $80-90 \%$ of uveal melanomas. However, mutations in these genes are mutually exclusive(Decatur et al., 2016; Van Raamsdonk et al., 2010), so that the absence of mutations in one implies, with high probability, the presence of driver mutations in the other. On the other extreme, the pair formed by TP53 and ATRX shows strong, mutual positive conditionality in low-grade gliomas, such that driver substitutions in ATRX appear almost exclusively in mutated TP53 backgrounds(Liu et al., 2012).

We compared the results of Coselens with those obtained using a mutual information approach (SELECT), which was also developed to identify conditional selection in cancer(Mina et al., 2017). The comparison indicates that Coselens detects many more conditionally selected gene pairs than SELECT, but the two approaches seem to capture complementary aspects of conditional selection in cancer genomes (Figure 1d). By leveraging information from copy number alterations, SELECT can detect conditional selection in genes whose role in cancer depends on amplification and/or deletion, albeit at the cost of not providing easily interpretable effect sizes. In contrast, Coselens proves substantially more powerful at detecting and quantifying conditional selection among cancer genes that harbor driver substitutions, with 7- and 4-fold increases in the number of positive and negative conditional pairs compared to SELECT (Supplementary Figure 1). Such higher detection power can be partly explained by Coselens' ability to analyze genes that fail some of the pre-filtering criteria applied by SELECT (this is the case for 79 of the 250 Coselens-exclusive pairs).

\section{Mutational load does not substantially confound Coselens' estimates of conditional selection}

Previous studies have noted that detection of mutual exclusivity can be confounded by intrinsic differences in the frequency and spectrum of mutations across cancer subtypes(Kim et al., 2017; van de Haar et al., 2019). To minimize this risk, Coselens independently fits two baseline mutation models, one for samples with coding mutations in the split gene and another for samples without such 
mutations. The use of subset-specific models for the expected number of substitutions helps prevent spurious correlations resulting from across-subset variations in mutational signatures. However, differences in mutational load can also affect the detection of conditional selection indirectly, in cases when mutation availability is a limiting factor in tumor evolution. If differences in mutational load significantly affect the availability of mutations on which selection can act, then, associations between rare cancer genes could reflect patterns of differential mutation availability rather than conditional selection. To account for that possibility, we adopted two alternative correction methods: one applies sampling theory to assess the likelihood that each significant gene pair can be explained by differences in mutation availability, and the other uses regression to identify biases in the number of significant pairs that correlate with mutation load (see Methods). The results obtained with both methods were in close agreement, leading to the conclusion that $>80 \%$ of conditional gene pairs could not be explained by differential mutation availability (Figure 1c).

We also investigated whether nonsynonymous passenger mutations in split genes quantitatively affected the inference of conditional selection. Because conditional selection is inferred by partitioning tumors into those with and without coding mutations in one of the genes, the inclusion of non-driver mutants in the former set could lead to underestimating the real magnitude of interactions between driver mutations. To test whether estimates of conditional selection are indeed affected by passenger coding mutations in the split gene, we used Bayes theorem to derive an equivalence expression that relates the standardized effect size of conditional selection upon swapping the split and query genes (see Methods). This equivalence should hold exactly in the case when conditional selection results from epistasis between driver mutations, but deviations are expected to appear if the inference of conditional selection is biased by passenger coding mutations in the split gene. Following this rationale, we compared the standardized effect sizes of conditional selection in reciprocal gene pairs (Figure 1e). The close agreement between empirical estimates and theoretical predictions (intraclass correlation $=0.88, \mathrm{~N}=78, \mathrm{p}<1 \mathrm{e}-4$ ) confirms that estimates of coding vs driver conditional selection are good approximations of the driver vs driver interactions that are relevant for cancer development.

Functional association and classification of epistatic interactions in conditionally selected gene pairs

To better characterize the mechanisms underlying conditional selection, we searched the STRING database for functional and physical interactions between the split- and query-gene products. Based on that search, we calculated an interaction enrichment ratio that quantifies, for any subset of gene pairs, the enrichment in predicted interactions of different types with respect to the set of all tested gene pairs. We found that enrichment in interactions becomes stronger with the increase of the effect size of conditional selection (Figure 2a). Pairs subject to significant conditional selection encompass more interactions than expected by chance, with a 1.5 -fold enrichment ( $95 \% \mathrm{Cl} 1.2-2.0)$ in the case of positive conditional pairs and a 4.2 -fold enrichment $(95 \% \mathrm{Cl} 2.7-6.6)$ in the case of negative conditional pairs (Figure $2 b$ ). Regarding the specific nature of interactions, no significant differences were found between functional and physical associations (that is, binding), although the latter seem to be slightly more prevalent among the negative conditional pairs.

Epistasis between cancer genes can be classified attending to the sign and magnitude of the conditional selection on driver substitutions. We identified 4 major classes of epistasis (Figure 2c): strict dependence, when driver (that is, positively selected) substitutions in the query gene are only 
observed in the presence of coding mutations in the split gene; facilitation, when driver substitutions are more likely to occur when the split gene is mutated; interference, when driver substitutions are less likely to occur when the split gene is mutated; and masking, when mutations in the split gene completely abolish the effect of driver substitutions in the query gene. Although strict dependence and masking appear as the extremes in the continuum that goes from facilitation to independence to interference, they can be distinguished by testing the null hypothesis that there is no significant selection on nonsynonymous mutations. By far, the most prevalent form of conditional selection is facilitation, which was identified in more than half of the significant gene pairs (Figure $2 \mathrm{~d}$ ). Focusing on the extreme classes, masking represents almost $40 \%$ of negative epistatic interactions ( $11 \%$ of the total), whereas strict dependence only represents $20 \%$ of positive interactions ( $14 \%$ of the total).

Positive and negative epistatic interactions differ in the way that reciprocal pairs relate to each other (Figure 2e). Negative epistasis tends to be symmetrical and often involves mutual masking. This is consistent with negative epistasis arising from functional redundancy although it could also reflect the existence of multiple, incompatible mutational paths to cancer (see below). On the other side, the spectrum of positive epistasis is broader and combines cases of strict interdependence and asymmetric facilitation. A biologically plausible interpretation of asymmetric facilitation is that driver mutations in a "master" cancer gene open new pathways of tumor development that involve the dependent gene. Supporting this interpretation, mutations in the master gene tend to show a higher degree of clonality (mean relative difference in variant allele frequency $=0.13, p=0.009$, 1-sample Student's t-test, $n=20$ ) and therefore are likely to precede mutations in the dependent gene (Figure 2f). In contrast, no systematic differences in clonality were found between genes that participate in symmetric epistasis. We observed that 6 of the 15 most asymmetric pairs identified in this study involved PTEN mutations in endometrial cancers (Supplementary Table 3), which underscores the fundamental role of PTEN inactivation as an early driver event in this cancer type(Levine et al., 1998; Zhang and $\mathrm{Yu}, 2010)$. Another highly asymmetric pair, observed in gastric and colorectal cancer, consists of TP53 as the master and RB1 as the dependent gene. From a functional perspective, the asymmetry of conditional selection between TP53 and RB1 suggests that an intact TP53 can partially compensate for the loss of RB1, but not vice versa.

\section{Conditional selection across cancer types}

Different cancer types broadly vary in the number of gene pairs subject to conditional selection, with the highest numbers found in endometrial and colorectal cancers, followed by stomach and bladder cancers (Figure 3a). To quantify the contribution of conditional selection to cancer somatic evolution, we compared the numbers of driver substitutions per tumor that were affected by conditional selection across cancer types (Figure $3 \mathrm{~b}$ ). We found that positive conditional selection often accounts for a sizable fraction (25-50\%) of all driver substitutions. The relative contributions of interference and masking are generally smaller although in some tumors, such as uveal melanoma, lower glade gliomas, and endometrial cancers, negative epistasis could lead to lack of selection for (and therefore hinder fixation of) $>40 \%$ potential driver mutations.

Both the number of gene pairs subject to conditional selection and the average number of conditionally-selected driver substitutions strongly correlate with the total number of driver substitutions per tumor (Figure $3 c-d$ ). These associations persist when controlling for several possible confounding factors, including the number of samples and mutations per cancer type, median 
mutational load, and number of gene pairs tested, that might unequally affect our ability to detect conditional selection in different cancer types (Spearman partial correlation coefficients rho $=0.95$ $\left(p<10^{-8}\right)$ for the number of gene pairs and rho $=0.80\left(p<10^{-4}\right)$ for the number of conditional drivers, $n=22$ ). A strong correlation was observed also with the number of non-conditionally-selected driver substitutions (Spearman partial correlation coefficients rho $=0.96\left(p<10^{-8}\right)$ for the number of gene pairs and rho $=0.75(p<0.001)$ for the number of conditional drivers, $n=22)$. Taken together, these observations imply that conditional selection is intrinsic to cancer somatic evolution, its contribution becoming increasingly important as the number of driver mutations increases.

Most gene pairs subject to conditional selection are highly specific to a single cancer type, with only $3 \%$ positive and $12 \%$ negative conditional pairs found in $>1$ cancer type (Figure $3 \mathrm{~d}$ ). Such higher promiscuity of negative conditional gene pairs, though small in magnitude, is statistically significant (chi-square $=6.24, p=0.03$, permutation test). Not surprisingly, multi-tissue gene pairs include wellknown examples of genes that display mutually exclusive or concurrent mutation patterns in pancancer analyses(Kandoth et al., 2013). Among the negative conditional pairs found in multiple cancer types, those involving oncogenes KRAS/NRAS and BRAF are easily ascribed to the functional redundancy in activating the Ras signaling pathway. The mutual exclusivity between $A R I D 1 A$ and TP53 possibly results from functional codependency in suppressing tumor development through transcriptional regulation(Guan et al., 2011; Wu et al., 2014). However, the mechanisms underlying the negative conditionality between mutations in TP53 and KRAS (and also TP53 and PIK3CA) remain unclear and could reflect fundamental, but poorly understood interactions among major pathways involved in cancer development. Positive conditional pairs spanning multiple cancer types include associations among the genes ATRX, IDH1, and TP53, which are known to harbor concurrent mutations in brain tumors (glioblastoma and lower grade gliomas)(Brennan et al., 2013; Cancer Genome Atlas Research et al., 2015; Liu et al., 2012). Other multi-tissue pairs are TP53-RB1 (detected in bladder cancer and glioblastoma), ARID1A-ERBB3 (bladder and stomach cancer), and PGM5-RNF43 (colorectal and stomach cancer). Interestingly, the gene $P G M 5$ (encoding phosphoglucomutase-like protein 5) has not been previously reported as a cancer gene, although low PGM5 expression has been linked to poor survival in liver and colorectal cancers(Jiao et al., 2019; Sun et al., 2019). Almost 75\% (35 out of 47) coding mutations in PGM5 detected in stomach and colorectal cancers correspond to a single nonsynonymous substitution with predicted deleterious effect (SIFT: 0.01 - deleterious; PolyPhen: 0.573 - possibly damaging) affecting position 98 (Ile $>V$ al) of the encoded protein, without loss of the second copy of the gene. In contrast, this substitution only represents $7 \%$ (7 out of 96) PGM5 coding mutations in other cancer types (Figure 3f). Thus, the PGM5 p.198V mutation is likely to be a specific driver of gastric and colorectal cancers that possibly operates through a gain-offunction mechanism.

Apart from the broadly spread gene pairs described above, the specificity of most conditional pairs for a single cancer type suggests that epistasis is often context-dependent and is conditioned on the physiology of the tumor(Park and Lehner, 2015; Yeang et al., 2008). This context dependency is manifest at two levels: first, cancer genes involved in conditional selection are often specific to one or a small number of cancer (sub)types; second, when the same gene appears in more than one cancer type, it is often involved in different conditional pairs. The first situation is illustrated by HLA$C$, which we identified as a conditional cancer gene in BRAF-mutant colorectal cancer (Figure 4). Conditional selection on HLA-C inactivating mutations is probably mediated by the highly 
immunogenic nature of tumors of the consensus molecular subtype 1 (CMS-1), whereby the loss of MHC-I-mediated antigen presentation confers a selective advantage(Jhunjhunwala et al., 2021). Supporting this interpretation, multiple studies have described driver mutations in $B 2 M$ and HLA genes in CMS-1 colorectal cancer and other microsatellite unstable tumors characterized by high levels of CD8+ T cell infiltration(Castro et al., 2019; Kloor et al., 2007; Shukla et al., 2015). The second situation is exemplified by RNF43 and its different conditionality patterns observed in colorectal, gastric, and endometrial cancers (Figure 4 and Supplementary Figure 2). In agreement with these differences, two distinct mechanisms have been proposed to explain how the loss of RNF43 contributes to carcinogenesis, with deregulation of Wnt signaling being dominant in the colon and modulation of the DNA damage response playing the principal role in the stomach(Neumeyer et al., 2021; Neumeyer et al., 2019). An even more striking example is presented by conditionally selected mutations in KRAS and TP53, which are antagonistic in lung and endometrial tumors, but synergistic in pancreatic cancer. For the latter cancer type, recent research has shown that mutant KRAS and p53 interact through the CREB1 protein to promote metastasis(Kim et al., 2021). The pronounced cancer type-specificity of conditional selection among widespread cancer genes highlights the need to integrate functional pleiotropy, cellular environment, and tumor physiology in models of cancer evolution(DeGregori, 2017).

\section{Epistasis networks}

To further elucidate how the interactions among somatic mutations affects tumor evolution, we built epistasis networks for each cancer type by connecting the genes that are involved in conditional selection. The most salient feature of such networks is their modular organization (Figure 4). Modularity implies that cancer genes organize into clearly differentiated groups (hereafter modules), such that genes within a module connect to each other through positive epistatic interactions, whereas genes from different modules display negative or no interactions. Modularity is most evident in cancer types where conditional selection affects a large fraction of driver events, such as low-grade glioma, colorectal, and endometrial cancers.

Inspection of the genes that belonged to different modules suggests an association between the modular structure of the epistasis network and the existence of well-defined molecular cancer subtypes. This association is especially clear in low-grade gliomas, where the three main modules encompassing (i) IDH1, ATRX, and TP53; (ii) CIC and FUBP1; and (iii) EGFR and PTEN, correspond, respectively, to three recently proposed molecular subtypes based on the IDH1 mutation and $1 p / 19 q$ codeletion status(Cancer Genome Atlas Research et al., 2015; Ceccarelli et al., 2016), whereas negative conditional selection between the genes of the first module and the NF1 gene is reminiscent of the molecular landscape found in gliomas from patients with neurofibromatosis type 1 and the LGm6 subgroup of sporadic glioma(Ceccarelli et al., 2016; D'Angelo et al., 2019; Verhaak et al., 2010). In glioblastoma, positive conditional selection among IDH1, ATRX, and TP53 defines a module associated with G-CIMP (glioma-CpG island methylator phenotype) tumors from the proneural subtype(Brennan et al., 2013; Noushmehr et al., 2010). To a lesser extent, the high prevalence of mutations in BRAF suggests a connection between consensus molecular subtype 1 of colorectal cancer and a module involving BRAF, BMPR2, RNF43 and HLA-C(Guinney et al., 2015). Similarly, in endometrial cancer, the detection of positive conditional selection between TP53 and PPP2R1A and the antagonism between these two genes and PTEN are consistent with the mutational landscape of 
serous endometrial tumors of the "copy number-high" molecular subtype(Cancer Genome Atlas Research et al., 2013; McConechy et al., 2011).

A possible explanation for the association between conditionally selected gene sets and cancer subtypes could be that the latter are a confounding factor for the inference of conditional selection. More precisely, because all samples from the same cancer type are pooled for the analysis, groups of genes that are preferentially mutated in a single subtype but not in the rest could appear as being jointly selected(van de Haar et al., 2019). To rule out this possibility, we carried out a reanalysis of conditional selection in cancer types with sufficient sample size and well-defined molecular subtypes, stratifying the data according to subtype classification. Although the reduction in sample size affected the ability to detect conditional pairs as statistically significant, we recovered some of the key interactions that characterized the modules of the epistasis network (Supplementary Table 4). More generally, we found that the estimated effect sizes for conditional selection in individual subtypes are highly correlated with the values obtained in the whole dataset (Figure 5a). Altogether, the results of the stratified analysis show that conditional selection also occurs within molecular subtypes and, therefore, is not an artifact of the joint analysis of samples from different groups.

Mutual exclusivity and co-occurrence among cancer somatic mutations have traditionally been interpreted in terms of within-pathway redundancy and across-pathway synergy(Yeang et al., 2008). In the simplest scenario, such synergy involves activation of an oncogenic pathway that promotes proliferation coupled with inactivation of a tumor suppressor pathway that regulates a cell cycle checkpoint(Cui et al., 2007). A relevant question concerning across-pathway synergy of driver mutations is whether it occurs in a combinatorial (or gene-agnostic) manner, that is, regardless of the specific genes that are mutated in each pathway(Oikonomou et al., 2014). To address this question, we investigated the distribution of triangular motifs in the epistasis networks. If acrosspathway synergy was gene-agnostic, positive epistasis would manifest as an excess of triangular motifs containing two positive and one negative interaction (Figure 5b). Contrary to this expectation, we found a significant excess of triangular motifs containing one positive and two negative interactions in the epistasis networks (Figure $5 \mathrm{~d}$ ). Such configuration suggests a more complicated scenario, in which across-pathway synergy is gene-specific, that is, depends on which genes are mutated in each pathway (Figure 5c). Indeed, visual inspection of epistasis networks reveals a highly gene-specific pattern of across-pathway interactions, whereby genes from the same pathway (for example, $R N F 43$ and $A P C$, or $B R A F$ and $R A S$ ) rarely display positive interactions with the same partners.

To better understand the effect of epistasis on cancer mutation landscapes, we simulated tumor evolution in the presence of conditional selection (Figure 6). The simulation results suggest that the structure of epistasis networks can play a major role in determining the set of driver mutations observed in evolved tumors. In particular, highly modular facilitation networks with across-module interference, such as those observed in low grade gliomas, colorectal, and endometrial cancers, spontaneously give rise to clearly differentiated mutation-based subtypes. This phenomenon is less pronounced in star-like epistasis networks (that is, those characterized by a single master driver that enhances the fitness of several secondary drivers) and completely absent in negative-modular networks (those corresponding to an idealized scenario of full within-pathway redundancy and across-pathway synergy). 


\section{Conditional selection affects survival rates}

To assess the clinical relevance of conditional selection among cancer genes, we investigated the association between the modules of the epistasis network and patient survival times (Suppl Figure 3). To that end, we assigned tumor samples to epistatic modules by selecting, for each sample, the module that harbored the highest number of coding mutations. Then, we carried out a survival analysis using two methods, Cox regression and non-parametric log-rank tests. Regardless of the method, module assignment was significantly associated with differential survival in 5 cancer types (glioblastoma, lower grade glioma, head and neck squamous carcinomas, pancreas adenocarcinoma, and endometrial cancer). An association between conditional selection and differential survival was also observed at the gene pair level, although the statistical significance was lower due to the small sample sizes (Suppl Figure 4). Of the 80 gene pairs with sufficient number of samples (at least 10 double mutants and 10 single mutants for each gene), we identified 9 instances ( $11 \%$ of the pairs), in which patient survival differed between tumors carrying coding mutations in both genes and those mutated in each gene alone (Cox regression and log-rank tests with $p<0.1$ for the omnibus test and $\mathrm{p}<0.15$ for double- vs single-mutant comparisons). For comparison, we ran the same analysis on nonconditionally selected gene pairs, finding that only $4.6 \%$ (27 out of 580 ) showed differences in patient survival between double and single mutants. Compared to single-gene mutants, the survival of patients with double mutations can be improved, worsened, or unaffected. Notably, worse patient survival is associated with gene pairs subject to positive conditional selection (ARID1A-ERBB3 in bladder cancer, CASP8-FAT1 in head and neck cancers, FGFR2-TP53 in endometrial cancer, and MUC17-NF1 in melanoma), whereas improved patient survival is associated with negative conditional selection (PPP2R1A-PTEN and ARID1A-PPP2R1A in endometrial cancer). The association with better patient survival in PPP2R1A-PTEN and ARID1A-PPP2R1A double mutants holds when controlling for mutational load, and suggests that mutual exclusivity in these gene pairs could result from synthetic lethality. Likewise, synthetic lethality might underlie other instances of negative conditional selection that could not be tested due to a lack of double mutants, as has been shown for KRAS and EGFR in lung cancer(Unni et al., 2015).

\section{Limitations of the study}

An obvious limitation of the approach developed and applied in this work is that we only sought to identify pairwise interactions between driver mutations. Extending Coselens to search for higherorder interactions is straightforward. However, because quantifying conditional selection while accounting for heterogeneity in mutational mechanisms requires partitioning the data in as many groups as conditions, and the number of conditions grows exponentially with the interaction order, the detailed study of high-order interactions can only be performed in large datasets and for frequently mutated cancer genes.

Another limitation is that we only analyzed point mutations that lead to amino acid replacement. Extending the approach to other types of mutations, in particular Copy Number Variation (CNV), will require developing the corresponding mutational models that, in the case of $\mathrm{CNV}$, will have to take into account correlations among adjacent genes. Nevertheless, some predictions seem feasible: mutations in TP53 are often associated with cancer subtypes that display chromosome instability and frequent $\mathrm{CNV}$, which is consistent with the role of p53 in aborting division of cells with genomic 
1 alterations(Hanel and Moll, 2012; Motoyama and Naka, 2004). Thus, we expect that positive

2 selection on copy number variants is conditional to having inactivating mutations in the p53 pathway.

\section{Conclusions}

5 The results of this work expand the catalogue of conditionally selected pairs of cancer genes by 6 several folds and demonstrate wide spread and importance of conditional selection in cancer 7 evolution. Indeed, in some cancer types, up to $50 \%$ of driver mutations are involved in such epistatic 8 interactions. Furthermore, we observed association between conditional selection and patient 9 survival, attesting to the clinical relevance of epistasis. Perhaps, most notably, the findings of this 10 work emphasize the striking complexity of the epistatic interactions among driver mutations that is 11 manifest at several levels. First, we identified multiple cases of all theoretically conceivable forms of 12 epistatic interactions, both positive and negative, including strict dependence, facilitation, 13 interference, and masking. Second, many of the conditionally selected gene pairs are cancer subtype14 specific and organize into highly modular epistasis networks. Third, we found that synergistic 15 epistatic interactions between signaling pathways are highly gene-specific. Conceivably, epistatic interactions shape differentiated cancer subtypes such that early driver mutations define the subsequent evolutionary path. This evolutionary path of advanced tumors involves mutations in a specific set of additional genes comprising the modules in the epistasis network, and determines the key properties of a molecular cancer subtype. Accordingly, differences in the pervasiveness of conditional selection across cancer types could underlie the existence of clearly delineated cancer type-specific gene sets in some types, such as colorectal and endometrial cancers, and gliomas, but not in others, such as lung and liver cancers(Iranzo et al., 2018). The hypothesis that subtypes are shaped by conditional selection is consistent with previous findings(Zhang et al., 2017) and was further supported by our simulations results, which suggest that the formation of subtypes depends on the type of modularity in the epistasis network. Finally, the finding that conditional interactions involving widespread cancer genes are cancer type-dependent raises the possibility that the same gene might act through different mechanisms in different cancer subtypes. Such specificity could limit the applicability of gene-targeting therapies across cancer types. 


\section{Acknowledgements}

2 We thank Iñigo Martincorena and Koonin group members for helpful discussions. This work was 3 initiated during an internship of G.G. at Koonin group, which was possible thanks to the NIH Summer 4 Internship Program in Biomedical Research. This work utilized the computational resources of the 5 NIH HPC Biowulf cluster (http://hpc.nih.gov).

6 J.I. is supported by the Ramón y Cajal Programme of the Spanish Ministry of Science (Grant No. RYC7 2017-22524), the Agencia Estatal de Investigación of Spain (Grant No. PID2019-106618GA-I00), and 8 the Severo Ochoa Programme for Centres of Excellence in R\&D of the Agencia Estatal de Investigación 9 of Spain (Grant No. SEV-2016-0672 (2017-2021) to the CBGP). G.G. is supported by the $10 \mathrm{DHHS} / \mathrm{PHS} /$ National Institutes of Health (Grant No. 2R01DE019637-10, "Patterning the vertebrate 11 dentition through replacement and repair). J.C.-E. is supported by the Youth Employment Initiative 12 of the European Social Fund through a junior postdoctoral contract from Comunidad de Madrid 13 (Grant No. PEJD-2019-POST/BIO-16377). E.V.K. is supported by intramural research program funds 14 of the National Institutes of Health (National Library of Medicine).

15

\section{Author contributions}

17 J.I. and E.V.K. designed and supervised the study; J.I. and G.G. developed the coselens software and 18 generated the primary results; J.I., G.G., and E.V.K. analyzed the results; J.C.-E. developed and simulated the computational model of tumor evolution; J.I., G.G., J.C-E., and E.V.K. wrote the paper.

20

\section{Declaration of interests}

22 The authors declare no competing interests. 


\section{Figure legends}

Figure 1: Inference of pairwise conditional selection in cancer genes. a) Overview of the Coselens method. The effect size $\Delta N_{d}$ is the difference in the frequency of driver substitutions in a given gene (the query gene) between tumors that do and do not harbor coding mutations in a second gene (the split gene). b) Effect size distributions for statistically significant and not-significant conditional gene pairs. Negative effect sizes are represented by their absolute values. c) Number of gene pairs subject to significant conditional selection (FDR $\leq 0.05$ ), classified by the sign of their interaction. The shaded green bar $(*)$ indicates a small subset of significant positive pairs whose conditional mutation patterns can be explained by differences in mutation availability. d) Comparison of Coselens and SELECT(Mina et al., 2017) methods for the detection of conditionally selected gene pairs. CNA: copy number alterations. e) Comparison of the standardized effect sizes that result from reciprocally exchanging the query gene by the split gene (an outlier at [18.4, 8.7] is not shown). The symmetry of standardized effect sizes implies that passenger mutations in the split gene do not substantially affect the estimates of conditional selection acting on the query gene.

Figure 2: Functional association and epistasis classification of conditionally selected gene pairs. a) Protein-protein interactions between gene products of the same conditional pair. The STRING enrichment ratio ( $y$ axis) was calculated by pooling all gene pairs above a given intensity of conditional selection $\left(\Delta N_{d} \geq x ; \mathrm{x}\right.$ axis) and comparing their STRING interaction scores with a reference base value calculated using all possible gene pairs. The small arrow at $\Delta N_{d}=0.15$ indicates the effect size of conditional selection at which most interactions become statistically significant. Shaded regions correspond to $95 \%$ confidence intervals. The noisy values obtained for $\Delta N_{d}>0.4$ are due to the small number of gene pairs that reach such high levels of conditional selection. b) Enrichment in different types of protein-protein interactions, calculated for the 296 gene pairs subject to significant conditional selection (whiskers indicate $95 \%$ confidence intervals). c) Epistasis is quantified attending to the frequency of driver substitutions in the query gene when the split gene does not harbor coding mutations ( $x$ axis) and when it does ( $y$ axis). In the absence of epistasis, the frequency of drivers in the query gene is not affected by the mutation status of the split gene, resulting in a 1:1 trend. Facilitation and interference manifest as positive and negative deviations from that trend and their magnitude is quantified by the angle $\theta$ (normalized by a factor $\pi / 4)$. Strict dependence and masking correspond to the extreme cases $x=0(\theta=1)$ and $y=0$ $(\theta=-1)$, respectively. None of the negative data points in the figure significatively deviates from 0 . d) Relative abundances of different classes of epistasis among 296 significantly conditional gene pairs. e) Asymmetry of epistatic interactions. Each data point corresponds to a statistically significant conditional gene pair whose reciprocal pair (with split and query gene roles exchanged) is also significant. Purely symmetric interactions (whereby both genes equally affect selection on each other) lie on the diagonal. f) Mean relative difference in the variant allele frequencies for coding mutations in genes subject to reciprocal positive epistasis. Gene pairs were grouped based on the asymmetry of the epistatic interaction (low asymmetry if $\left|\theta_{A B}-\theta_{B A}\right|<0.2$; high asymmetry if $\left|\theta_{A B}-\theta_{B A}\right| \geq 0.2$ ). Boxes comprise the 25th-75th percentiles; thick lines and notches represent the median and its $95 \%$ confidence interval, respectively; whiskers extend to the last data point within 1.5 times the interquartile range; outliers beyond that range are represented as with the symbol + .

Figure 3: Distribution of conditionally selected gene pairs across cancer types. a) Number of gene pairs affected by significant conditional selection in each cancer type. Reciprocal gene pairs (with 
reciprocally exchanged split and query genes) are counted only once. No significant gene pairs were detected for sarcoma, thymoma, esophageal, renal (clear cell), liver, ovarian, and prostate cancers. b) Number of conditionally selected driver substitutions and total driver substitutions per tumor. Negative values can be interpreted as substitutions that could have been drivers in the absence of epistasis, but did not become established due to lack of selection. c, d) Association between the total number of driver substitutions per tumor ( $x$ axis) and (c) the number of significant gene pairs, (d) the number of conditionally selected driver substitutions ( $y$ axis). Each data point corresponds to a cancer type $(n=22)$. These associations remain highly significant after controlling for sample size (number of tumors and total mutations), median mutational load, and number of pairs tested. e) Conditional gene pairs found in multiple cancer types. f) Distribution of mutations along the PGM5 protein in stomach, colorectal, and other cancer types. The cartoon at the bottom indicates the location of alpha-D-phosphohexomutase, alpha/beta/alpha domains I, II, and III, with the predicted active site in red.

Figure 4: Epistasis networks based on conditional selection for some representative cancer types (see Suppl. Fig. 2 for the remaining cancer types). Nodes represent cancer genes involved in conditional selection; edges denote conditionally selected gene pairs. Cancer genes that are not involved in conditional selection are not represented, although they sometimes harbor a sizeable fraction of driver substitutions. Node size is proportional to the frequency of driver substitutions in a gene; edge color represents the sign of the interaction; edge width and transparency indicates the degree of evidence for an interaction as indicated in the legend. Reciprocal interactions are those detected as statistically significant regardless of which is the query and which is the split gene; non-reciprocal interactions are only detected as significant for a specific choice of split and query genes (either because the reverse choice is not significant or because it could not be tested due to insufficient sample size).

Figure 5: Conditional selection and structure of cancer molecular subtypes. a) Effect sizes of conditional selection at the cancer type ( $x$ axis) and subtype ( $y$ axis) levels. Each data point corresponds to a gene pair tested in at least one subtype. Gray lines represent the orthogonal least squares fit for each cancer type. b) Simple scenario of cancer progression through the alteration of two independent pathways (each represented in a gray rectangle), generating a pattern of withinpathway mutual exclusivity and across-pathway co-occurrence. c) A more complex scenario, in which cross-talk between pathways modifies the effect of alterations affecting other pathways. In this scenario, mutual exclusivity among functionally redundant mutations in the same pathway can still be expected; however, only some combinations of mutations affecting different pathways can act synergistically driving the progression of specific cancer subtypes. Scenarios (b) and (c) can be distinguished attending to the sign of triangular motifs in the epistasis network. d) Number of signed triangular motifs in the epistasis networks of colon cancer (COAD), endometrial cancer (UCEC), and all cancer types. Gray bars correspond to randomized networks with the same size and signed degree distributions obtained by pairwise rewiring of the network edges, and serve as a null model for motif counts (error bars represent $95 \%$ confidence intervals based on 2000 randomizations). BRCA: breast cancer; LGG: low-grade glioma.

Figure 6: Modular epistasis in simulated networks lead to differentiation of cancer genomes into mutational subtypes. a) Tumor evolution was simulated in the presence of conditional selection, with epistatic interactions determined by networks with different degrees of modularity. For each 
bioRxiv preprint doi: https://doi.org/10.1101/2022.01.10.475617; this version posted January 11,2022 . The copyright holder for this preprint

(which was not certified by peer review) is the author/funder. This article is a US Government work. It is not subject to copyright under 17 USC 105 and is also made available for use under a CCO license.

1 network, the mutation composition of 500 simulated tumors was explored using principal 2 component analysis to search for mutational differentiation into subtypes. b) Conditional selection 3 can generate clearly delineated mutational subtypes if the underlying epistasis network (top) is 4 characterized by intra-module facilitation and inter-module interference. The bottom panels show 5 the results of a principal component analysis for each network, with each point representing a 6 simulated tumor. 


\section{STAR Methods}

\section{Dataset and data preprocessing}

Somatic mutation calls for The Cancer Genome Atlas (TCGA) dataset, generated by the Multi-Center Mutation Calling in Multiple Cancers project(Ellrott et al., 2018), were downloaded from the Genomic Data Commons repository of the National Institutes of Health (https://gdc.cancer.gov/aboutdata/publications/mc3-2017). Clinical and survival data for TCGA were also downloaded from the Genomic Data Commons repository of the National Institutes of Health (https://gdc.cancer.gov/node/905/; file TCGA-CDR-SupplementalTableS1.xlsx)(Liu et al., 2018). To avoid the confounding effect of purifying selection, which is non- negligible in hypermutated tumors(Persi et al., 2018), samples with $>3000$ coding mutations were classified as hypermutators and removed from subsequent analyses.

\section{Inference of conditional selection: the Coselens method}

To quantify conditional selection at the gene level, we developed Coselens (COnditional SELection on the Excess of Nonsynonymous Substitutions), a tool that makes extensive use of the $d n d s c v$ R package (dndSCV, RRID:SCR_017093) to quantify selection in cancer and normal tissues(Martincorena et al., 2017). We briefly describe the fundamentals of the $d n d s c v$ method, as long as it is needed to understand the modifications introduced by Coselens; we refer the reader to the original publication for further details. In short, $d n d s c v$ estimates the number of missense and truncating (nonsense and essential splice site) substitutions per gene that would be expected in the absence of selection by combining a nucleotide substitution model with 192 rates (12 possible substitutions in each of the 16 possible trinucleotide contexts) and a set of genomic covariates, which greatly improve the performance of the method at low mutation loads. The model parameters are estimated through negative binomial regression on the number of synonymous mutations to maximize the following likelihood function:

$$
\mathcal{L}\left(\boldsymbol{r}, \boldsymbol{t}, \alpha, \beta ; \boldsymbol{n}_{s}^{o b s}\right)=\prod_{g, j} P_{P o i s s o n}\left(n_{s, g, j}^{o b s} ; \lambda=t_{g} r_{j} L_{s, g, j}\right) P_{G a m m a}\left(t_{g} ; \alpha, \beta\right)
$$

where $n_{s, g, j}^{o b s}$ is the number of synonymous substitutions observed in the gene $g$ and the index $j$ corresponds to the substitution classes (192 by default although substitution models with fewer or more classes can be easily accommodated), $r_{j}$ is the mutation rate for each substitution class, $L_{s, g, j}$ is the number of sites in gene $g$ that are synonymous under the substitution class $j$, and $t_{g}$ is a factor accounting for variable mutation rates across genes, which is assumed to follow a gamma distribution with parameters $\alpha$ and $\beta$. Once those parameters have been inferred, the expected number of nonsynonymous substitutions in the absence of selection is obtained by replacing $L_{s, g, j}$ by the number of sites in gene $g$ that are susceptible to missense and truncating substitutions $\left(L_{m, g, j}\right.$ and $L_{t, g, j}$, respectively). The selection parameter $\omega_{k, g}$ is then calculated for each gene as the ratio between the observed number of mutations and their neutral expectation $\left(\omega_{k, g}=\sum_{j} n_{k, g, j}^{o b s} /\right.$ $\sum_{j} n_{k, g, j}^{e x p}$, where the subscript $k \in\{m, t\}$ refers to missense and truncating substitutions). From the perspective of the likelihood function, the selection parameter is a multiplicative factor in $\lambda$ that modifies the observed substitution frequency with respect to the neutral expectation, with $\omega_{k, g}>1$ and $\omega_{k, g}<1$ implying positive and purifying selection, respectively. The significance of $\omega_{k, g}$ is 
computed through a likelihood ratio test, where the null and alternative hypotheses correspond to $\omega_{k, g}=1$ and $\omega_{k, g} \neq 1$, respectively.

To develop Coselens, we modified the dndscv method in two substantial ways. First, we quantified the differences (rather than the ratios) between the observed number of missense and truncating substitutions and their neutral expectations:

$$
\Delta n_{m, g}=\frac{\sum_{j} n_{m, g, j}^{o b s}-\sum_{j} n_{m, g, j}^{e x p}}{T} ; \Delta n_{t, g}=\frac{\sum_{j} n_{t, g, j}^{o b s}-\sum_{j} n_{t, g, j}^{e x p}}{T}
$$

where $T$ is the number of samples used in the analysis. Missense and truncating substitution excesses result from mutations that have reached detectable frequencies through positive selection. Because the effect of purifying selection in non-hypermutator tumors is negligible(Martincorena et al., 2017; Persi et al., 2018), the total excess of nonsynonymous mutations in a gene, $\Delta n_{g}=\Delta n_{m, g}+\Delta n_{t, g}$, is a good estimate of the number of driver substitutions $N_{d}$ in that gene (formally, $N_{d}=$ $\left((-1) n_{m, g}^{\text {exp }}+(-1) n_{t, g}^{\text {exp }}\right) / T=\Delta n_{g}$; note that we omitted the subscript $g$ in $N_{d}$ for simplicity although it is a gene-specific quantity). Second, we modified the likelihood ratio test to allow for comparison of $\Delta n_{g}$ between two sets of samples. To this end, Coselens separately fits the $d n d s c v$ substitution model to each sample group (hereafter $X$ and $Y$ ), obtaining two sets of parameters for the neutral scenario $(\boldsymbol{\theta}(X)$ and $\boldsymbol{\theta}(Y))$ and two sets of nonsynonymous mutation excesses $\left(\Delta n_{k, g}(X)\right.$ and $\Delta n_{k, g}(Y)$, with $\left.k \in\{m, t\}\right)$. The likelihood ratio test is built according to the following null and alternative hypotheses (2 degrees of freedom):

$$
\begin{aligned}
& \mathrm{H}_{0}: \Delta n_{m, g}(X)=\Delta n_{m, g}(Y) ; \Delta n_{t, g}(X)=\Delta n_{t, g}(Y) \\
& \mathrm{H}_{1}: \Delta n_{m, g}(X) \neq \Delta n_{m, g}(Y) ; \Delta n_{t, g}(X) \neq \Delta n_{t, g}(Y)
\end{aligned}
$$

To restrict the hypothesis test to differences in the number of drivers, but not in the underlying mutation rates, the null hypothesis does not introduce any constraint on the parameters $\boldsymbol{\theta}(X)$ and $\boldsymbol{\theta}(Y)$. Accordingly, the likelihood of the null hypothesis is evaluated using group-specific parameters for each observation and joint nonsynonymous mutation excesses $\Delta n_{m, g}(X+Y)$ and $\Delta n_{t, g}(X+Y)$ ), for which the general expression is

$$
\Delta n_{k, g}(X+Y)=\frac{\Delta n_{k, g}(X) T(X)+\Delta n_{k, g}(Y) T(Y)}{T(X)+T(Y)} ; k \in\{m, t\}
$$

Here, $T(X)$ and $T(Y)$ are the numbers of samples in each group. The joint nonsynonymous excesses can be incorporated in the likelihood function by leveraging the relationship between the mutation excess $\Delta n_{k, g}$ and the mutation ratio $\omega_{k, g}$, that is, $\omega_{k, g}=1+\Delta n_{k, g} T / n_{k, g}^{e x p}$. Under the null hypothesis, this relationship becomes

$$
\omega_{k, g}\left(X \mid \mathrm{H}_{0}\right)=1+\frac{\Delta n_{k, g}(X+Y) T(X)}{n_{k, g}^{\exp }(X)} ; \quad \omega_{k, g}\left(Y \mid \mathrm{H}_{0}\right)=1+\frac{\Delta n_{k, g}(X+Y) T(Y)}{n_{k, g}^{\text {exp }}(Y)}
$$

Thus, the likelihood of the null hypothesis becomes

$$
\mathcal{L}\left(\mathrm{H}_{0}\right)=\mathcal{L}\left(\mathrm{H}_{0}, \boldsymbol{\theta}(X) ; \boldsymbol{n}_{k, g}^{o b s}(X)\right) \mathcal{L}\left(\mathrm{H}_{0}, \boldsymbol{\theta}(Y) ; \boldsymbol{n}_{k, g}^{o b s}(Y)\right)
$$




$$
\mathcal{L}\left(\mathrm{H}_{0}, \boldsymbol{\theta} ; \boldsymbol{n}_{k, g}^{o b s}\right)=\prod_{k, j} P_{\text {Poisson }}\left(n_{k, g, j}^{o b s} ; \lambda=t_{g} r_{j} L_{k, g, j} \omega_{k, g}\left(\cdot \mid \mathrm{H}_{0}\right)\right) P_{G a m m a}\left(t_{g} ; \alpha, \beta\right)
$$

The expression for the likelihood of the alternative hypothesis is analogous, except that the $\omega_{k, g}$ parameters are calculated using sample-specific mutation excesses, that is,

$$
\omega_{k, g}\left(X \mid \mathrm{H}_{1}\right)=1+\frac{\Delta n_{k, g}(X) T(X)}{n_{k, g}^{\exp }(X)} ; \quad \omega_{k, g}\left(Y \mid \mathrm{H}_{1}\right)=1+\frac{\Delta n_{k, g}(Y) T(Y)}{n_{k, g}^{\exp }(Y)}
$$

The output of the likelihood ratio test is the probability that the excess of nonsynonymous substitutions (missense, truncating, or both) in a given gene differs between the two sets of samples. As mentioned above, in the context of nearly absent purifying selection, such as in cancer evolution, the effect size can be interpreted as the difference in the number of driver mutations per sample in the gene of interest:

$$
\Delta N_{d}=N_{d}(X)-N_{d}(Y)=\Delta n_{m, g}(X)+\Delta n_{t, g}(X)-\Delta n_{m, g}(Y)-\Delta n_{t, g}(Y)
$$

In our case, the sets $X$ and $Y$ correspond to samples with and without coding mutations in a second gene; however, the Coselens method is general and can be applied to compare any pair of sample sets (e.g. to study differential selection across tissues or clinical conditions). Although we focused on genes under positive selection, Coselens can also detect differences in purifying selection if the excess of substitutions is negative. To achieve the highest accuracy, Coselens should be run on whole exome data or targeted sequencing data including as many genes as possible, as that boosts the performance of parameter inference. The output can be subsequently filtered to restrict false discovery rate correction and downstream analysis to a set of genes of interest.

We provide Coselens as an open-source $\mathrm{R}$ package that can be downloaded from https://github.com/ggruenhagen3/coselens. Besides the main method described above, the package includes some extensions to study the excess of small indels and test for differences in missense or truncating substitutions only.

\section{Conditional selection within cancer types}

To investigate pairwise conditional selection among cancer genes, we compiled a set of candidate gene pairs, to which we would apply the Coselens method. Each candidate gene pair consisted of a split gene (the gene inducing conditional selection) and a query gene (the gene that experiences conditional selection). Candidate gene pairs were separately identified in each cancer type; in that sense, one can think of gene pairs as triplets (cancer type - split gene - query gene). Given a split gene, conditional selection was assessed by dividing the samples into those that carried coding mutations (nonsynonymous substitutions and small indels) in the split gene and those that do not, comparing both groups with Coselens, and filtering the results according to the list of query genes associated to that split gene.

A preliminary set of query genes was built by finding genes evolving under significant positive selection in each cancer type with the R package dndscv (default parameters). From this preliminary set, we built the set of split genes that harbored coding mutations in at least 25 samples, while being free of coding mutations in at least another 25 samples. Then, we expanded the set of query genes by running Coselens and searching for conditional selection on known cancer genes not included in 
the preliminary set of query genes. For this exploratory step, we considered a list of 369 cancer genes from previous studies(Iranzo et al., 2018; Martincorena et al., 2017) and applied the BenjaminiHochberg false discovery rate correction to all possible pairs consisting of a split gene and one of those known cancer genes. This approach identified 20 additional query genes, for which positive selection is only detectable in a restricted subset of samples (e.g., HLA-C and PGM5 in BRAF-mutant colorectal cancer). We finally studied conditional selection on all possible split - query gene pairs within each cancer type, applying a false discovery rate correction to the pooled results (that is, jointly considering all cancer types). Additionally, to capture cases of reciprocal conditionality among gene pairs that could have been missed due to insufficient statistical power, we applied restricted hypothesis testing to split-query gene combinations whose reciprocal had been found significant under the standard approach.

\section{Negative control}

A control dataset was built by randomly assigning mock "mutated split gene" and "wild-type split gene" labels to each tumor. Relabeling was done such that the total number of mutations in the control subsets remained approximately the same as in the original ones. Specifically, for each cancer type, tumor samples were randomly chosen and assigned to the "mutated split gene" subset until the total number of mutations in the group reached or surpassed the number of mutations in the original subset of samples with mutations in the split gene. The reason to keep the number of mutations (rather than the number of samples) per group fixed is that Coselens pools together all mutations from all samples within a group. By keeping that number approximately unchanged, we ensured that the sensitivity of Coselens was similar in the negative control and in the original dataset. Furthermore, it is important to perform the randomization at the sample (rather than mutation) level to conserve the structure of within-sample correlations, so that the model covariates remain informative. Note that, because this randomization procedure removes systematic biases in mutational load and subtype composition between subsets, it is not suitable to assess the rate of false positives induced by such confounding factors. The entire workflow described in the previous section was repeated for 40 randomized control datasets, finding an average of 6.5 significant pairs per run $(0.1 \%$ of the tested pairs). The comparison between this number and the value found in the original dataset (296 significant pairs) yields an empirical false discovery rate of $\sim 2 \%$.

\section{Conditional selection within cancer subtypes}

Samples from colorectal cancer were classified into molecular subtypes, and each subtype was separately analyzed as described above. The consensus molecular subtypes of colorectal cancer samples were obtained from the data repository of the colorectal cancer subtyping consortium (https://www.synapse.org/\#!Synapse:syn2623706/files/)(Guinney et al., 2015). After removing hypermutated tumors (>3000 coding mutations), only 49 samples remained in the CMS1 group. Therefore, the sample size threshold to consider a candidate gene as a split gene in CMS1 was lowered to at least 18 samples with and 18 samples without coding mutations in that gene. A similar subtype-based analysis was applied to endometrial cancer, low-grade glioma, and breast cancer. The molecular subtype classification for those samples was obtained from the original publications(Cancer Genome Atlas Research et al., 2013; Ceccarelli et al., 2016) and the PanCancer Atlas dataset(Berger et al., 2018) of the cBioPortal repository(Cerami et al., 2012). 
Gene pairs were classified by epistasis modes attending to the variation in the excess of nonsynonymous mutations in the first gene $(A)$ when the second gene $(B)$ does or does not harbor coding mutations $\left(N_{d}(A \mid B)\right.$ and $N_{d}(A \mid \bar{B})$, respectively). First, we tested whether $N_{d}(A \mid B)$ or $N_{d}(A \mid \bar{B})$ were significantly distinct from zero. Within the dndscv framework, testing $N_{d} \neq 0$ is equivalent to testing whether the ratio $d n / d s \neq 0$. To account for multiple comparisons, we applied the Benjamini-Hochberg correction to all pairs tested for conditional selection and set the significance threshold to a false discovery rate of $5 \%$. Next, we classified conditionally selected gene pairs in one of the following categories: (i) masking epistasis, if $N_{d}(A \mid \bar{B})>N_{d}(A \mid B)=0$; (ii) interference, if $N_{d}(A \mid \bar{B})>N_{d}(A \mid B)>0$; (iii) facilitation, if $N_{d}(A \mid B)>N_{d}(A \mid \bar{B})>0$; (iv) strict dependence, if $N_{d}(A \mid B)>N_{d}(A \mid \bar{B})=0$; (v) strict dependence with sign change, if $N_{d}(A \mid B)>0>$ $N_{d}(A \mid \bar{B})$; (vi) compensation, if $N_{d}(A \mid \bar{B})<N_{d}(A \mid B)=0$; (vii) conditional deleterious, if $N_{d}(A \mid B)>$ $N_{d}(A \mid \bar{B})=0$; and (viii) incompatible, if $N_{d}(A \mid \bar{B})>0>N_{d}(A \mid B)$. Because the test for conditional selection can be more sensitive than the test for $N_{d} \neq 0$, there were some cases where $N_{d}(A \mid B) \neq$ $N_{d}(A \mid \bar{B})$ but neither $N_{d}(A \mid B)$ nor $N_{d}(A \mid \bar{B})$ were significantly different from zero. Those cases were left unclassified.

\section{Estimation of the number of driver substitutions affected by conditional selection}

For a given cancer type and a split gene $B$, the average number of driver events per tumor that involve conditional selection on the query gene $A$ was obtained as $\Delta N_{d}(A, B) X_{B}$, where $\Delta N_{d}(A, B)=$ $N_{d}(A \mid B)-N_{d}(A \mid \bar{B})$ is the effect size of conditional selection and $X_{B}$ is the fraction of tumors with coding mutations in the split gene $B$. To estimate the total number of driver events affected by conditional selection, we summed over all directed pairs $(A, B)$ that were significant with $\mathrm{FDR} \leq 5 \%$, including reciprocal pairs that were significant under restricted hypothesis testing.

\section{Estimation of the number of driver mutations per patient}

Although the number of driver mutations can be estimated within the dndscv (and Coselens) framework, we chose an independent approach to minimize the risk that correlations with the number of conditional driver substitutions were affected by the presence of uncontrolled confounding factors. Therefore, we implemented a previously described simple regression-based approach(Iranzo et al., 2018). The number of driver mutations per patient was estimated as the intercept of a linear regression model that had, as independent variables, the cancer type, the number of coding mutations in passenger genes, and the interaction of both; and as the dependent variable, the number of coding mutations in 441 significantly mutated cancer genes (the union set of those previously detected(Martincorena et al., 2017) and those found in this study).

\section{Functional association between genes in conditionally selected pairs}

Each split-query gene pair was searched against the STRING database of protein interactions (v11.0). If an interaction was found in the database, the pair was assigned a score $s$ equal to the STRING score divided by 1000 (this normalization accounted for the fact that STRING scores range from 0 to 1000 , with higher values representing higher confidence in the predicted interaction). Pairs not found in the STRING database were assigned a score of zero. Given a subset $G$ of gene pairs, the STRING interaction ratio was calculated as $r_{G}=\sum_{G} s / \sum_{G}(1-s)$. In the special case when $s$ takes a binary value (1 or 0 ), $r_{G}$ becomes the ratio between the number of gene pairs that are known to interact and those for which there is no evidence of interaction. The STRING interaction ratio was computed 
for increasing magnitudes of conditional selection by defining a nested collection of subsets $G_{x}$, with $0<x<\max \left(\Delta N_{d}\right)$, such that each subset includes all gene pairs whose effect size $\Delta N_{d}$ is greater than $x$ (accordingly, $G_{0}$ corresponds to the set of all gene pairs included in the analysis). The interaction enrichment ratio for a given effect size was then calculated as $R\left(\Delta N_{d}\right)=r_{G_{\Delta N_{d}}} / r_{G_{0}}$. Conceptually, the enrichment ratio is analogous to a risk ratio, with the difference that the response variable is continuous rather than binary. Following Pagano and Gauvreau (2000), the 95\% confidence interval was calculated as $C I_{95}\left(\Delta N_{d}\right)=\exp (\ln (R) \pm 1.96 \times S E(\ln (R)))$, where $S E(\ln (R))=\sqrt{\frac{1}{\sum_{G_{\Delta N_{d}}} s}+\frac{1}{\sum_{G_{\Delta N_{d}}}(1-s)}-\frac{1}{\sum_{G_{0}} s}-\frac{1}{\sum_{G_{0}}(1-s)}}$.

\section{Reciprocity of conditional selection}

Let $P(X \mid \bar{Y})$ be the conditional probability that query gene $X$ harbors a driver mutation provided that there are no driver mutations in the split gene $Y$. Conversely, let $P(X \mid Y)$ be the conditional probability that the query gene $X$ harbors a driver mutation provided that there are driver mutations in the split gene $Y$. Then, the magnitude of epistasis-driven conditional selection can be quantified as the difference $\Delta P(X, Y)=P(X \mid Y)-P(X \mid \bar{Y})$. For any pair of genes $A$ and $B$, we are interested in finding an expression that relates $\Delta P(A, B)$ and $\Delta P(B, A)$, that is, the magnitude of conditional selection when $A$ is the query gene and $B$ is the split gene, and when the query and split roles are reversed. Combining Bayes theorem and the identity $P(X)+P(\bar{X})=1$, the difference $\triangle P(A, B)$ can be expressed as

$$
\Delta P(A, B)=P(A \mid B)-P(A \mid \bar{B})=P(A)\left(\frac{P(B \mid A)}{P(B)}-\frac{1-P(B \mid A)}{1-P(B)}\right)=\frac{P(A)(P(B \mid A)-P(B))}{P(B)(1-P(B))}
$$

where $P(A)$ and $P(B)$ are the marginal probabilities for finding driver mutations in genes $A$ and $B$, respectively. In a similar way, $\Delta P(B, A)$ can be written as

$$
\Delta P(B, A)=P(B \mid A)-P(B \mid \bar{A})=P(B \mid A)-\frac{P(B)-P(B \mid A) P(A)}{1-P(A)}=\frac{P(B \mid A)-P(B)}{1-P(A)}
$$

Extracting $P(B \mid A)-P(B)$ from both expressions, and after some algebraic manipulation, we obtain

$$
\frac{\Delta P(A, B)}{P(A)(1-P(A))}=\frac{\Delta P(B, A)}{P(B)(1-P(B))}
$$

If presence or absence of driver mutations in genes $A$ and $B$ are modeled as independent Bernoulli random variables with success probabilities $P(A)$ and $P(B)$, the denominators in eq. (1) correspond to the variances of such random variables across tumors. Accordingly, the left and right terms of eq. (1) can be interpreted as the standardized effect sizes of conditional selection for the query genes $A$ and $B$, respectively. Eq. (1) implies that, if conditional selection results from epistasis between driver mutations, the standardized effect sizes should be equal for reciprocal query-split gene pairs.

To compare this theoretical prediction with the empirical estimates, we first established a correspondence between the marginal and conditional probabilities from eq. (1) and the nonsynonymous mutation excesses calculated from cancer genomic data. To that end, we assumed that only the first driver substitution in each cancer gene is positively selected, and therefore the 
probability to find a driver mutation in a given gene can be approximated by the mean number of driver substitutions per tumor in that gene. Although this assumption is strictly valid only for singlehit oncogenes, it also holds for tumor suppressor genes, as long as the inactivation of the second copy of the gene can occur via large deletions. Thus, to validate eq. (1) with empirical genomic data, we replaced $\Delta P(A, B) \approx \Delta N_{d}(A, B)$ and $P(A) \approx N_{d}(A \mid B) \times X_{B}+N_{d}(A \mid \bar{B}) \times\left(1-X_{B}\right)$, where $X_{B}$ is the fraction of tumors with coding mutations in the split gene. This second approximation potentially could become a major source of error because the empirical split of tumor samples is based on the presence of coding mutations, whereas conditionality in the theoretical expression specifically involves driver mutations. Therefore, calculating the goodness of fit of the empirical data to the theoretical expectation in eq. (1) is a straightforward way to assess whether the empirical estimates are robust with respect to the use of coding mutations rather than driver mutations in the split gene to quantify conditional selection.

\section{Assessment of the effect of differential mutation availability on conditional selection estimates}

To investigate whether conditional selection can be explained by differences in mutation availability, two alternative approaches were adopted. The first approach consisted of inferring, given the expected number of mutations per tumor in the query gene, the probability that at least one driver mutation becomes available for selection to act on. We considered a Poisson distribution for the number of substitutions per gene per tumor, such that given a local mutation rate $\mu$, the probability that at least one driver mutation becomes available in the gene of interest is $\pi(\mu)=1-\exp (b \mu)$. Given a set of $T$ independent tumors, the number of tumors in which the driver mutation becomes available $(k)$ follows a binomial distribution with parameters $T$ and $\pi(\mu)$. Following the central limit theorem, for large enough $T$, the fraction of tumors in which the driver mutation becomes available $(f=k / T)$, can be approximated by a normal distribution with mean $\pi(\mu)$ and variance $\pi(\mu)(1-\pi(\mu)) / T$. Therefore, given two sets of values for $f, \mu$, and $T$ (corresponding to tumors with and without mutations in the split gene), it is possible to apply a maximum likelihood approach to infer the only unknown parameter of the model $(b)$ and then obtain the probability that differences in $f$ can be explained by changes in $\mu$. To perform these calculations, we took the expected number of non-synonymous and non-sense mutations per sample per gene inferred with the dndscv method as a gene-wise measure of $\mu$; and the average number of drivers per gene per tumor as a gene-wise measure of $f$ (i.e., $f \approx N_{d}$, following the same rationale described above for the analysis of reciprocity). A continuity correction was included when assessing the likelihood, such that $L(b ; f, \mu$, $T)=\operatorname{NormCDF}(f+0.5 / T)-\operatorname{NormCDF}(f-0.5 / T)$, where "NormCDF" is the cumulative probability function for a normal distribution with mean $\pi(\mu)$ and variance $\pi(\mu)(1-\pi(\mu)) / T$. For each conditional gene pair, the probability that it can be explained by differential mutation availability was calculated as the product $p=p_{1} p_{2}$, where $p_{1}\left(f_{1}, \mu_{1}, T_{1}\right)$ is the probability that $f_{1}$ (the average number of drivers per tumor in samples with a mutated split gene) comes from a normal distribution with mean $\pi\left(\mu_{1}\right)$ and variance $\pi\left(\mu_{1}\right)\left(1-\pi\left(\mu_{1}\right)\right) / T_{1}$ and $p_{2}\left(f_{2}, \mu_{2}, T_{2}\right)$ represents the same probability for samples without coding mutations in the split gene. In the case of reciprocal interactions, we combined the probabilities of the direct and reverse pairs $\left(p^{A B}\right.$ and $p^{B A}$, respectively) as $p=p^{A B} p^{B A}$. The resulting probabilities were corrected for multiple comparisons with FDR $=5 \%$. 
The second approach involved a regression analysis to assess how the excess of gene pairs displaying positive epistasis (compared to those that display negative epistasis) changes with the difference in the median mutational load of tumors with and without mutations in the split gene. By focusing on the difference between positive and negative epistatic pairs, rather than on the total numbers, we accounted for the fact that mutational load has a directed effect on mutation availability, that is, higher (respectively lower) mutational loads increase (decrease) mutation availability, resulting in an apparent excess of positive (negative) epistatic pairs. We found a weak, but significant association between the difference in mutational load and the excess of positive epistatic pairs in which a split gene is involved ( 0.16 pairs per every 100 mutations/exome difference in samples with and without coding mutations in the split gene; ANCOVA stratified by cancer types, $R^{2}=0.15 ; p=1 e-9$ ). To assess how this association could translate into apparent (mutation availability-driven) conditional selection, we adopted a Bayesian approach to infer the probability that a pair involving a given split gene can be attributed to differences in mutational load. First, we modeled the prior for the number of such pairs as a Poisson distribution with mean $\lambda=a \times \Delta \mu_{g}$, where $a$ is the slope of the regression model and $\Delta \mu_{g}$ is the difference in the median mutational load of tumors that do and do not harbor mutations in the split gene of interest. To obtain the posterior distribution, we took into account the total number of conditional interactions observed for that split gene, $k_{g}$, by truncating the Poisson distribution between 0 and $k_{g}$. The expected contribution of differential mutation availability to the number of conditional pairs was then calculated as the mean of the truncated distribution.

\section{Detection of modules in the epistasis networks}

Positive conditional modules in the epistasis networks were identified with SiMap, a community detection algorithm that takes into account the sign and weight of interactions(Esmailian and Jalili, 2015). We used the effect sizes $\Delta N_{d}(A, B)$ as interaction weights and preprocessed the networks to extract their connected components before running the community detection algorithm with default parameters. Overlapping modules were identified by adding, for each gene, the weights of its interactions with genes from other communities. A secondary module assignation was made for those genes for which the total weight of interactions with the original community was $<1.5$ times the total weight of interactions with the secondary module. Samples were assigned to the module with which they shared the greatest number of mutated genes. Samples without mutations in genes from the epistasis network were assigned to a null module. Ambiguous cases (samples with the same number of genes mutated in $>1$ module) were resolved only if they met the following two conditions: (i) there were only 2 candidate modules, to which the sample could be assigned, and (ii) the fraction of mutated genes belonging to each module was different. If both conditions were met, the sample was assigned to the module with the largest fraction of mutated genes; otherwise, the module assignation was considered ambiguous.

\section{Survival analysis}

We conducted a survival analysis to investigate if the combination of mutations in two conditionally selected genes affected patient survival, compared to mutations in a single gene. For each gene pair $(A, B)$ in a given cancer type, tumor samples were split in 3 groups: (i) those with coding mutations in gene $A$ but not in gene $B$, (ii) those with coding mutations in gene $B$ but not in $A$, and (iii) those with coding mutations in both genes (samples without mutations in $A$ or $B$ were not included). Gene pairs that did not reach a minimum of 10 samples in each group and 100 samples overall were no further 
considered. For those gene pairs that reached sufficient sample size, we adopted two approaches to assess the statistical significance of differences in survival between the double-mutation group and each of the single-mutation groups: (a) Kaplan-Meier estimation followed by non-parametrical logrank tests(Clark et al., 2003), and (b) Cox regression analysis with the double-mutation group as the reference category(Bradburn et al., 2003). In both cases, we assessed the overall significance (omnibus test for the null hypothesis that survival is the same in the three groups) and the significance of the differences between each single-gene mutant and the double mutant. We reported the cases with $p<0.1$ for the omnibus test and $p<0.15$ for both single vs double mutant comparisons. Statistical analyses were carried out with the $\mathrm{R}$ packages survminer and survival(Therneau and Grambsch, 2000).

The association between patient survival and positive epistatic modules with $>15$ samples was assessed using the same approaches, and the cancer types reported in the text are those with $p<0.05$ for the omnibus tests.

\section{Comparison of Coselens and SELECT methods}

The list of conditionally selected events in the TGCA identified using the SELECT algorithm was collected from the Supplementary Information of the original publication(Mina et al., 2017). To reconcile the fact that SELECT operates at the level of "selected functional events" (SFE) and Coselens operates at the gene level, each SFE was mapped to the gene or genes affected by that SFE. Pairs of conditional SFE that mapped to the same gene (typically associated with double inactivation of tumor suppressor genes) were not considered. Directionality was ignored and the significance threshold of both methods was adjusted to ensure a FDR $<10 \%$, which was the value originally used by the developers of SELECT when applying it to the TCGA dataset.

\section{Simulation of tumor evolution}

The evolution of a population of (pre)cancer cells was modeled as a discrete time birth-and-death process, where death also accounted for differentiation into non-proliferative lineages. At each time step, each cell could either die (with probability $P_{d}$ ) or divide (with probability $1-P_{d}$ ). Following previous work by McFarland et al(McFarland et al., 2013), the death probability was set to

$$
P_{d}=\frac{D(N)}{B\left(d_{i}, d_{e}, p\right)+D(N)}
$$

where $D(N)=N / K$ represents density-dependent death rate ( $N$ is the current population size and parameter $K$ determines the equilibrium population size in the absence of mutations). The birth term was modeled as

$$
B\left(d_{i}, d_{e}, p\right)=\frac{\left(1+s_{d}\right)^{d_{i}}\left(1+\epsilon s_{d}\right)^{d_{e}}}{\left(1+s_{p}\right)^{p}}
$$

where $s_{p}$ is the fitness effect of a passenger mutation, $s_{d}$ is the fitness effect of a driver mutation in the absence of epistasis, and $\epsilon$ is an epistasis coefficient that accounts for the increase in the fitness effect of a driver mutation due to positive epistasis. The variable $p$ represents the number of passenger mutations in the genome of a given cell, $d_{i}$ is the number of driver mutations that are not affected by epistasis, and $d_{e}$ is the number of driver mutations affected by positive epistasis. We 
1 assumed a scenario, in which negative epistasis fully masks the effect of driver mutations, and

2 therefore, driver mutations affected by negative epistasis do not contribute to the birth term.

3 Upon division, one of the daughter cells can acquire driver and passenger mutations. The number of

4 new driver and passenger mutations was drawn from Poisson distributions with the means $T_{d} \mu$ (for

5 drivers) and $T_{p} \mu$ (for passengers), where $T_{d}$ and $T_{p}$ are the mutation target sizes for drivers and

6 passengers, respectively. New driver mutations were randomly assigned to the cancer genes from

7 the epistasis network; if a gene had already been hit by a previous driver mutation, all subsequent

8 driver mutations in that gene were treated as neutral.

9 To study the effect of epistasis in cancer genome evolution, we simulated 5 scenarios represented 10 by the following epistasis networks: (i) no epistasis, represented as a "network" of 10 disconnected 11 genes; (ii) $2 \times 5$-node star networks, with positive epistasis between the central and peripheral genes 12 of each star; (iii) $2 \times 5$-node star networks as in (ii), adding negative epistasis between the central 13 genes of each star; (iv) $2 \times 5$-node clique networks, with positive epistasis among all gene pairs from 14 the same clique, and negative epistasis affecting half of all possible gene pairs from different cliques; 15 and (v) $3 \times 5$-node "negative clique" network, with negative epistasis among all gene pairs from the 16 same clique, and positive epistasis affecting $10 \%$ of all possible gene pairs from different cliques (this 17 percentage was chosen to achieve a positive-to-negative interaction ratio that inversely mirrored the 18 synergistic clique scenario).

19 Simulations started from a clonal population of size $K$ with one driver mutation in a randomly 20 selected gene and no passengers. We used the following parameter values: $K=1000, \mu=10^{-8}$, $21 T_{d}=700, T_{p}=5 \times 10^{6}, s_{p}=10^{-3}, \epsilon=5$. To facilitate comparisons, the parameter $s_{d}$ was 22 separately set in each scenario, such that the average fitness effect when considering epistasis was 23 the same $\left(\left\langle s_{d}\right\rangle=0.1\right)$. Thus, in the disconnected and star networks (scenarios i-iii), we set $s_{d}=$ $24\left\langle s_{d}\right\rangle=0.1$, in the clique network (scenario iv) $s_{d}=2\left\langle s_{d}\right\rangle /(1+\epsilon) \approx 0.03$, and in the negative clique network (scenario v) $s_{d}=3\left\langle s_{d}\right\rangle / \epsilon=0.06$. Simulations ended when the cell population doubled its initial size, became extinct, or after 30,000 generations. The final composition of those populations that doubled the initial size (representing tumor progression) was used to investigate differentiation into mutation-based subtypes by running a principal component analysis on the mutation composition matrix that represented the most frequent genotypes of 500 independently evolving populations. 


\section{Supplemental Information}

Supplementary Figure 1 (related to Figure 1d): Comparison of Coselens and SELECT methods for the detection of conditionally selected gene pairs, separately showing the overlap of both methods for positive and negative epistasis. CNA: copy number alterations.

Supplementary Figure $\mathbf{2}$ (related to Figure 4): Epistasis networks for cancer types not shown in Figure 4. Nodes represent cancer genes involved in conditional selection; edges denote conditionally selected gene pairs. Cancer genes that are not involved in conditional selection are not represented, although they sometimes harbor a sizeable fraction of driver substitutions. Node size is proportional to the frequency of driver substitutions in a gene; edge color represents the sign of the interaction; edge width and transparency indicates the degree of evidence for an interaction as indicated in the legend. Reciprocal interactions are those detected as statistically significant regardless of which is the query and which is the split gene; non-reciprocal interactions are only detected as significant for a specific choice of split and query genes (either because the reverse choice is not significant or because it could not be tested due to insufficient sample size).

Supplementary Figure 3: Kaplan-Meier curves showing significant association between the modules of the epistasis network and patient survival times. Tumor samples were assigned to the module with which they shared the greatest number of mutated genes. Samples without mutations in genes from the epistasis network were assigned to a null module (e.g., GBM-0). P-values correspond to the logrank test for the null hypothesis that there are no differences among the three curves. GBM: glioblastoma; LGG: lower grade glioma; PAAD: pancreatic cancer; UCEC: endometrial cancer; HNSC: head and neck squamous carcinoma. Genes included in module GBM-1: ATRX, IDH1, RB1, TP53; GBM2: PTEN; LGG-1: ATRX, IDH1, TP53; LGG-2: CIC, FUBP1; LGG-3: EGFR, PTEN; LGG-4: IDH2; LGG-5: NF1; PAAD-1: KRAS, TP53; UCEC-1: ARID1A, CTCF, CTNNB1, INPPL1, JAK1, KANSL1, KMT2B, KMT2D, KRAS, NFE2L2, PIK3R1, PTEN, RB1, RNF43, TAF1, ZFHX3, ATM, CCND1, FGFR2, UPF3A; UCEC-5: PIK3CA; UCEC-6: PPP2R1A, TP53; HNSC-1: CASP8, CTCF, EP300, EPHA2, FAT1, FBXW7, HLA-A, HLA-B, HRAS, RAC1, TGFBR2; HNSC-2: CDKN2A.p14arf, CDKN2A.p16INK4a, TP53.

Supplementary Figure 4: Kaplan-Meier curves showing association between conditional selection at the gene pair level and differential survival. The $p$-value indicated by $p_{\text {all }}$ corresponds to the log-rank test for the null hypothesis that there are no differences among the three survival curves; $p_{1}$ and $p_{2}$ correspond to pairwise comparisons between the double mutant and each of the single mutants (logrank test).

Supplementary Table 1: Genes under significant levels of positive selection in at least one cancer type, used as "query genes" in this study. For each gene, the selection parameters for missense, nonsense, and indels, and the estimated number of driver mutations per tumor are shown. The table also indicates if a query gene was additionally considered as a "split gene".

Supplementary Table 2: Gene pairs under significant conditional selection in at least one cancer type, sorted by effect size. For each pair, the table shows the mean number of driver substitutions per tumor in the query gene in the presence ( $\mathrm{Nd}$.with) and absence ( $\mathrm{Nd}$.without) of coding mutations in the split gene, the magnitude of conditional selection (DeltaNd), the dependence index (theta), the epistasis class, and the q-values for the null hypotheses $\mathrm{Nd}$. with $=0, \mathrm{Nd}$. without $=0$, and DeltaNd $=$ 0 . 
1 Supplementary Table 3: Gene pairs that display reciprocal conditional selection, sorted by 2 asymmetry. For each pair, the table shows the dependence indices in both directions (theta_AB and 3 theta_BA), the asymmetry, the sign of epistasis, and the mean relative difference in variant allele 4 frequency.

5 Supplementary Table 4: Analysis of conditional selection within cancer subtypes for breast cancer 6 (BRCA), colorectal cancer (COAD), endometrial cancer (UCEC), and lower grade glioma (LGG). 7 Statistical significance levels are coded as n.s. (not significant), ${ }^{*}(p<0.05),{ }^{* *}(p<0.01)$, and ${ }^{* * *}$ $8 \quad(p<0.01)$. 


\section{References}

Ashworth, A., Lord, C. J., and Reis-Filho, J. S. (2011). Genetic interactions in cancer progression and treatment. Cell 145, 30-38.

Babur, O., Gonen, M., Aksoy, B. A., Schultz, N., Ciriello, G., Sander, C., and Demir, E. (2015). Systematic identification of cancer driving signaling pathways based on mutual exclusivity of genomic alterations. Genome biology 16, 45.

Berger, A. C., Korkut, A., Kanchi, R. S., Hegde, A. M., Lenoir, W., Liu, W., Liu, Y., Fan, H., Shen, H., Ravikumar, V., et al. (2018). A Comprehensive Pan-Cancer Molecular Study of Gynecologic and Breast Cancers. Cancer cell 33, 690-705 e699.

Bradburn, M. J., Clark, T. G., Love, S. B., and Altman, D. G. (2003). Survival analysis part II: multivariate data analysis--an introduction to concepts and methods. British journal of cancer 89 , 431-436.

Brennan, C. W., Verhaak, R. G., McKenna, A., Campos, B., Noushmehr, H., Salama, S. R., Zheng, S., Chakravarty, D., Sanborn, J. Z., Berman, S. H., et al. (2013). The somatic genomic landscape of glioblastoma. Cell 155, 462-477.

Cancer Genome Atlas Research, N., Brat, D. J., Verhaak, R. G., Aldape, K. D., Yung, W. K., Salama, S. R., Cooper, L. A., Rheinbay, E., Miller, C. R., Vitucci, M., et al. (2015). Comprehensive, Integrative Genomic Analysis of Diffuse Lower-Grade Gliomas. The New England journal of medicine 372, 24812498.

Cancer Genome Atlas Research, N., Kandoth, C., Schultz, N., Cherniack, A. D., Akbani, R., Liu, Y., Shen, H., Robertson, A. G., Pashtan, I., Shen, R., et al. (2013). Integrated genomic characterization of endometrial carcinoma. Nature 497, 67-73.

Cannataro, V. L., Gaffney, S. G., and Townsend, J. P. (2018). Effect Sizes of Somatic Mutations in Cancer. J Natl Cancer Inst 110, 1171-1177.

Castro, A., Ozturk, K., Pyke, R. M., Xian, S., Zanetti, M., and Carter, H. (2019). Elevated neoantigen levels in tumors with somatic mutations in the HLA-A, HLA-B, HLA-C and B2M genes. BMC Med Genomics 12, 107.

Ceccarelli, M., Barthel, F. P., Malta, T. M., Sabedot, T. S., Salama, S. R., Murray, B. A., Morozova, O., Newton, Y., Radenbaugh, A., Pagnotta, S. M., et al. (2016). Molecular Profiling Reveals Biologically Discrete Subsets and Pathways of Progression in Diffuse Glioma. Cell 164, 550-563.

Cerami, E., Gao, J., Dogrusoz, U., Gross, B. E., Sumer, S. O., Aksoy, B. A., Jacobsen, A., Byrne, C. J., Heuer, M. L., Larsson, E., et al. (2012). The cBio cancer genomics portal: an open platform for exploring multidimensional cancer genomics data. Cancer Discov 2, 401-404.

Clark, T. G., Bradburn, M. J., Love, S. B., and Altman, D. G. (2003). Survival analysis part I: basic concepts and first analyses. British journal of cancer 89, 232-238.

Cui, Q., Ma, Y., Jaramillo, M., Bari, H., Awan, A., Yang, S., Zhang, S., Liu, L., Lu, M., O'ConnorMcCourt, M., et al. (2007). A map of human cancer signaling. Mol Syst Biol 3, 152.

D'Angelo, F., Ceccarelli, M., Tala, Garofano, L., Zhang, J., Frattini, V., Caruso, F. P., Lewis, G., Alfaro, K. D., Bauchet, L., et al. (2019). The molecular landscape of glioma in patients with Neurofibromatosis 1 . Nature medicine 25, 176-187. 
Decatur, C. L., Ong, E., Garg, N., Anbunathan, H., Bowcock, A. M., Field, M. G., and Harbour, J. W. (2016). Driver Mutations in Uveal Melanoma: Associations With Gene Expression Profile and Patient Outcomes. JAMA Ophthalmol 134, 728-733.

DeGregori, J. (2017). Connecting Cancer to Its Causes Requires Incorporation of Effects on Tissue Microenvironments. Cancer research 77, 6065-6068.

Di Micco, R., Fumagalli, M., Cicalese, A., Piccinin, S., Gasparini, P., Luise, C., Schurra, C., Garre, M., Nuciforo, P. G., Bensimon, A., et al. (2006). Oncogene-induced senescence is a DNA damage response triggered by DNA hyper-replication. Nature 444, 638-642.

Ellrott, K., Bailey, M. H., Saksena, G., Covington, K. R., Kandoth, C., Stewart, C., Hess, J., Ma, S., Chiotti, K. E., McLellan, M., et al. (2018). Scalable Open Science Approach for Mutation Calling of Tumor Exomes Using Multiple Genomic Pipelines. Cell Syst 6, 271-281 e277.

Esmailian, P., and Jalili, M. (2015). Community Detection in Signed Networks: the Role of Negative ties in Different Scales. Scientific reports 5, 14339.

Garraway, L. A., and Lander, E. S. (2013). Lessons from the cancer genome. Cell 153, 17-37.

Greaves, M., and Maley, C. C. (2012). Clonal evolution in cancer. Nature 481, 306-313.

Guan, B., Wang, T. L., and Shih le, M. (2011). ARID1A, a factor that promotes formation of SWI/SNFmediated chromatin remodeling, is a tumor suppressor in gynecologic cancers. Cancer research 71, 6718-6727.

Guinney, J., Dienstmann, R., Wang, X., de Reynies, A., Schlicker, A., Soneson, C., Marisa, L., Roepman, P., Nyamundanda, G., Angelino, P., et al. (2015). The consensus molecular subtypes of colorectal cancer. Nature medicine 21, 1350-1356.

Hanahan, D., and Weinberg, R. A. (2000). The hallmarks of cancer. Cell 100, 57-70.

Hanel, W., and Moll, U. M. (2012). Links between mutant p53 and genomic instability. J Cell Biochem 113, 433-439.

Iranzo, J., Martincorena, I., and Koonin, E. V. (2018). Cancer-mutation network and the number and specificity of driver mutations. Proceedings of the National Academy of Sciences of the United States of America 115, E6010-E6019.

Jerby-Arnon, L., Pfetzer, N., Waldman, Y. Y., McGarry, L., James, D., Shanks, E., Seashore-Ludlow, B., Weinstock, A., Geiger, T., Clemons, P. A., et al. (2014). Predicting cancer-specific vulnerability via data-driven detection of synthetic lethality. Cell 158, 1199-1209.

Jhunjhunwala, S., Hammer, C., and Delamarre, L. (2021). Antigen presentation in cancer: insights into tumour immunogenicity and immune evasion. Nature reviews Cancer 21, 298-312.

Jiao, Y., Li, Y., Jiang, P., Han, W., and Liu, Y. (2019). PGM5: a novel diagnostic and prognostic biomarker for liver cancer. PeerJ 7, e7070.

Kandoth, C., McLellan, M. D., Vandin, F., Ye, K., Niu, B., Lu, C., Xie, M., Zhang, Q., McMichael, J. F., Wyczalkowski, M. A., et al. (2013). Mutational landscape and significance across 12 major cancer types. Nature 502, 333-339.

Kim, M. P., Li, X., Deng, J., Zhang, Y., Dai, B., Allton, K. L., Hughes, T. G., Siangco, C., Augustine, J. J., Kang, Y. a., et al. (2021). Oncogenic KRAS recruits an expansive transcriptional network through mutant p53 to drive pancreatic cancer metastasis. Cancer Discovery, candisc.1228.2020. 
Kim, Y. A., Madan, S., and Przytycka, T. M. (2017). WeSME: uncovering mutual exclusivity of cancer drivers and beyond. Bioinformatics 33, 814-821.

Kloor, M., Michel, S., Buckowitz, B., Ruschoff, J., Buttner, R., Holinski-Feder, E., Dippold, W., Wagner, R., Tariverdian, M., Benner, A., et al. (2007). Beta2-microglobulin mutations in microsatellite unstable colorectal tumors. Int J Cancer 121, 454-458.

Leiserson, M. D., Vandin, F., Wu, H. T., Dobson, J. R., Eldridge, J. V., Thomas, J. L., Papoutsaki, A., Kim, Y., Niu, B., McLellan, M., et al. (2015). Pan-cancer network analysis identifies combinations of rare somatic mutations across pathways and protein complexes. Nat Genet 47, 106-114.

Levine, R. L., Cargile, C. B., Blazes, M. S., van Rees, B., Kurman, R. J., and Ellenson, L. H. (1998). PTEN mutations and microsatellite instability in complex atypical hyperplasia, a precursor lesion to uterine endometrioid carcinoma. Cancer research 58, 3254-3258.

Liu, J., Lichtenberg, T., Hoadley, K. A., Poisson, L. M., Lazar, A. J., Cherniack, A. D., Kovatich, A. J., Benz, C. C., Levine, D. A., Lee, A. V., et al. (2018). An Integrated TCGA Pan-Cancer Clinical Data Resource to Drive High-Quality Survival Outcome Analytics. Cell 173, 400-416 e411.

Liu, X. Y., Gerges, N., Korshunov, A., Sabha, N., Khuong-Quang, D. A., Fontebasso, A. M., Fleming, A., Hadjadj, D., Schwartzentruber, J., Majewski, J., et al. (2012). Frequent ATRX mutations and loss of expression in adult diffuse astrocytic tumors carrying IDH1/IDH2 and TP53 mutations. Acta Neuropathol 124, 615-625.

Martincorena, I., Raine, K. M., Gerstung, M., Dawson, K. J., Haase, K., Van Loo, P., Davies, H., Stratton, M. R., and Campbell, P. J. (2017). Universal Patterns of Selection in Cancer and Somatic Tissues. Cell.

Matlak, D., and Szczurek, E. (2017). Epistasis in genomic and survival data of cancer patients. PLoS computational biology 13, e1005626.

McConechy, M. K., Anglesio, M. S., Kalloger, S. E., Yang, W., Senz, J., Chow, C., Heravi-Moussavi, A., Morin, G. B., Mes-Masson, A. M., Australian Ovarian Cancer Study, G., et al. (2011). Subtypespecific mutation of PPP2R1A in endometrial and ovarian carcinomas. J Pathol 223, 567-573.

McFarland, C. D., Korolev, K. S., Kryukov, G. V., Sunyaev, S. R., and Mirny, L. A. (2013). Impact of deleterious passenger mutations on cancer progression. Proceedings of the National Academy of Sciences of the United States of America 110, 2910-2915.

Mina, M., Raynaud, F., Tavernari, D., Battistello, E., Sungalee, S., Saghafinia, S., Laessle, T., SanchezVega, F., Schultz, N., Oricchio, E., and Ciriello, G. (2017). Conditional Selection of Genomic Alterations Dictates Cancer Evolution and Oncogenic Dependencies. Cancer cell 32, 155-168 e156.

Misale, S., Di Nicolantonio, F., Sartore-Bianchi, A., Siena, S., and Bardelli, A. (2014). Resistance to anti-EGFR therapy in colorectal cancer: from heterogeneity to convergent evolution. Cancer Discov $4,1269-1280$.

Motoyama, N., and Naka, K. (2004). DNA damage tumor suppressor genes and genomic instability. Current opinion in genetics \& development 14, 11-16.

Neumeyer, V., Brutau-Abia, A., Allgauer, M., Pfarr, N., Weichert, W., Falkeis-Veits, C., Kremmer, E., Vieth, M., Gerhard, M., and Mejias-Luque, R. (2021). Loss of RNF43 Function Contributes to Gastric Carcinogenesis by Impairing DNA Damage Response. Cell Mol Gastroenterol Hepatol 11, 1071-1094. 
Neumeyer, V., Grandl, M., Dietl, A., Brutau-Abia, A., Allgauer, M., Kalali, B., Zhang, Y., Pan, K. F., Steiger, K., Vieth, M., et al. (2019). Loss of endogenous RNF43 function enhances proliferation and tumour growth of intestinal and gastric cells. Carcinogenesis 40, 551-559.

Nik-Zainal, S., Van Loo, P., Wedge, D. C., Alexandrov, L. B., Greenman, C. D., Lau, K. W., Raine, K., Jones, D., Marshall, J., Ramakrishna, M., et al. (2012). The life history of 21 breast cancers. Cell 149, 994-1007.

Noushmehr, H., Weisenberger, D. J., Diefes, K., Phillips, H. S., Pujara, K., Berman, B. P., Pan, F., Pelloski, C. E., Sulman, E. P., Bhat, K. P., et al. (2010). Identification of a CpG island methylator phenotype that defines a distinct subgroup of glioma. Cancer cell 17, 510-522.

Oikonomou, E., Koustas, E., Goulielmaki, M., and Pintzas, A. (2014). BRAF vs RAS oncogenes: are mutations of the same pathway equal? Differential signalling and therapeutic implications. Oncotarget 5, 11752-11777.

Pagano, M., and Gauvreau, K. (2000). Principles of biostatistics, 2nd edn (Pacific Grove, CA: Duxbury).

Park, S., and Lehner, B. (2015). Cancer type-dependent genetic interactions between cancer driver alterations indicate plasticity of epistasis across cell types. Mol Syst Biol 11, 824.

Persi, E., Wolf, Y. I., Leiserson, M. D. M., Koonin, E. V., and Ruppin, E. (2018). Criticality in tumor evolution and clinical outcome. Proceedings of the National Academy of Sciences of the United States of America 115, E11101-E11110.

Senft, D., Leiserson, M. D. M., Ruppin, E., and Ronai, Z. A. (2017). Precision Oncology: The Road Ahead. Trends Mol Med 23, 874-898.

Shukla, S. A., Rooney, M. S., Rajasagi, M., Tiao, G., Dixon, P. M., Lawrence, M. S., Stevens, J., Lane, W. J., Dellagatta, J. L., Steelman, S., et al. (2015). Comprehensive analysis of cancer-associated somatic mutations in class I HLA genes. Nature biotechnology 33, 1152-1158.

Srihari, S., Singla, J., Wong, L., and Ragan, M. A. (2015). Inferring synthetic lethal interactions from mutual exclusivity of genetic events in cancer. Biol Direct 10, 57.

Stratton, M. R., Campbell, P. J., and Futreal, P. A. (2009). The cancer genome. Nature 458, 719-724.

Sun, Y., Long, H., Sun, L., Sun, X., Pang, L., Chen, J., Yi, Q., Liang, T., and Shen, Y. (2019). PGM5 is a promising biomarker and may predict the prognosis of colorectal cancer patients. Cancer Cell Int 19, 253.

Therneau, T. M., and Grambsch, P. M. (2000). Modeling survival data : extending the Cox model, (New York: Springer).

Unni, A. M., Lockwood, W. W., Zejnullahu, K., Lee-Lin, S. Q., and Varmus, H. (2015). Evidence that synthetic lethality underlies the mutual exclusivity of oncogenic KRAS and EGFR mutations in lung adenocarcinoma. Elife 4, e06907.

van de Haar, J., Canisius, S., Yu, M. K., Voest, E. E., Wessels, L. F. A., and Ideker, T. (2019). Identifying Epistasis in Cancer Genomes: A Delicate Affair. Cell 177, 1375-1383.

Van Raamsdonk, C. D., Griewank, K. G., Crosby, M. B., Garrido, M. C., Vemula, S., Wiesner, T., Obenauf, A. C., Wackernagel, W., Green, G., Bouvier, N., et al. (2010). Mutations in GNA11 in uveal melanoma. The New England journal of medicine 363, 2191-2199. 
1 Verhaak, R. G., Hoadley, K. A., Purdom, E., Wang, V., Qi, Y., Wilkerson, M. D., Miller, C. R., Ding, L.,

2 Golub, T., Mesirov, J. P., et al. (2010). Integrated genomic analysis identifies clinically relevant

3 subtypes of glioblastoma characterized by abnormalities in PDGFRA, IDH1, EGFR, and NF1. Cancer

4 cell $17,98-110$.

5 Vogelstein, B., Papadopoulos, N., Velculescu, V. E., Zhou, S., Diaz, L. A., Jr., and Kinzler, K. W. (2013).

6 Cancer genome landscapes. Science 339, 1546-1558.

7 Wang, X., Fu, A. Q., McNerney, M. E., and White, K. P. (2014). Widespread genetic epistasis among 8 cancer genes. Nature communications 5, 4828.

9 Wappett, M., Dulak, A., Yang, Z. R., Al-Watban, A., Bradford, J. R., and Dry, J. R. (2016). Multi-omic 10 measurement of mutually exclusive loss-of-function enriches for candidate synthetic lethal gene 11 pairs. BMC genomics $17,65$.

12 Weghorn, D., and Sunyaev, S. (2017). Bayesian inference of negative and positive selection in 13 human cancers. Nat Genet 49, 1785-1788.

14 Wilkins, J. F., Cannataro, V. L., Shuch, B., and Townsend, J. P. (2018). Analysis of mutation, selection, 15 and epistasis: an informed approach to cancer clinical trials. Oncotarget 9, 22243-22253.

16 Williams, M. J., Werner, B., Heide, T., Curtis, C., Barnes, C. P., Sottoriva, A., and Graham, T. A.

17 (2018). Quantification of subclonal selection in cancer from bulk sequencing data. Nat Genet 50, 18 895-903.

19 Wu, R. C., Wang, T. L., and Shih le, M. (2014). The emerging roles of ARID1A in tumor suppression.

20 Cancer Biol Ther 15, 655-664.

21 Yeang, C. H., McCormick, F., and Levine, A. (2008). Combinatorial patterns of somatic gene

22 mutations in cancer. FASEB J 22, 2605-2622.

23 Zhang, H., Deng, Y., Zhang, Y., Ping, Y., Zhao, H., Pang, L., Zhang, X., Wang, L., Xu, C., Xiao, Y., and Li, 24 X. (2017). Cooperative genomic alteration network reveals molecular classification across 12 major 25 cancer types. Nucleic acids research 45, 567-582.

26 Zhang, S., and Yu, D. (2010). PI(3)king apart PTEN's role in cancer. Clin Cancer Res 16, 4325-4330. 
a

split gene B

query gene $A$

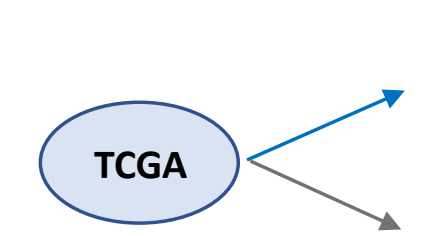

stratified by

cancer type

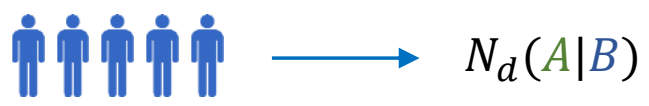
mutated

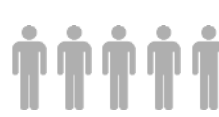

not mutated

dndscv

$N_{d}(A \mid \bar{B})$

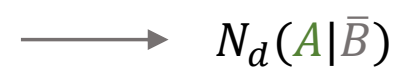

d

$\Delta N_{d}^{A, B}$
Figure 1

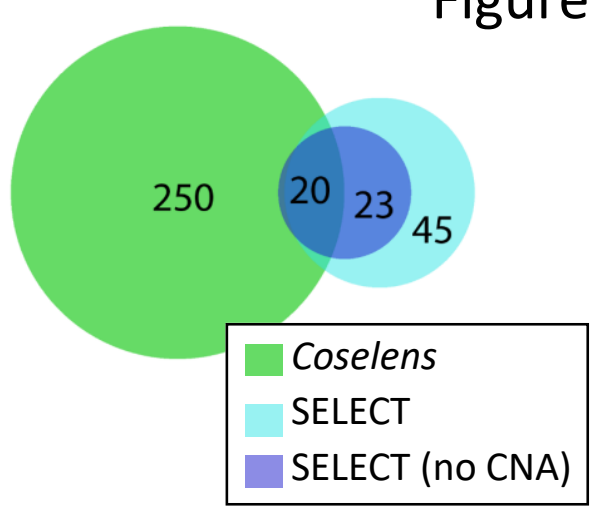

C

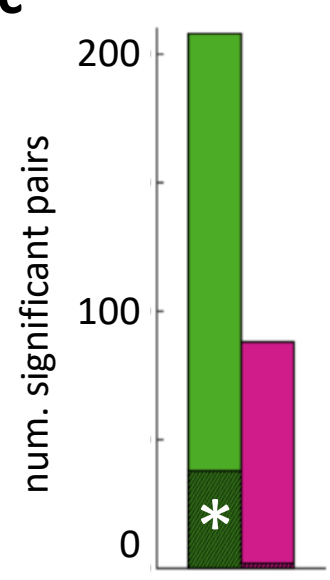

e

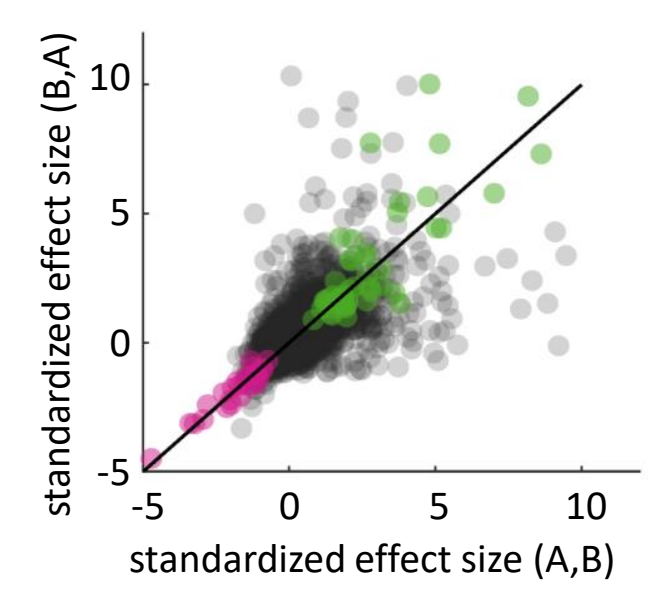

b

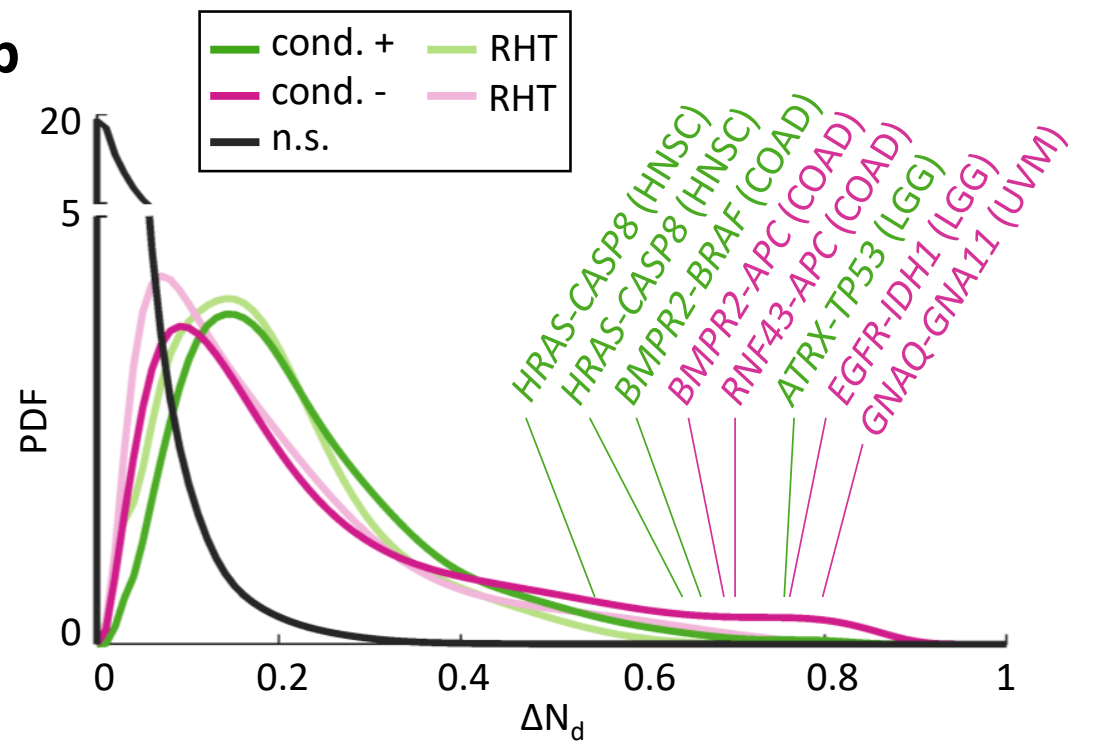


$a$

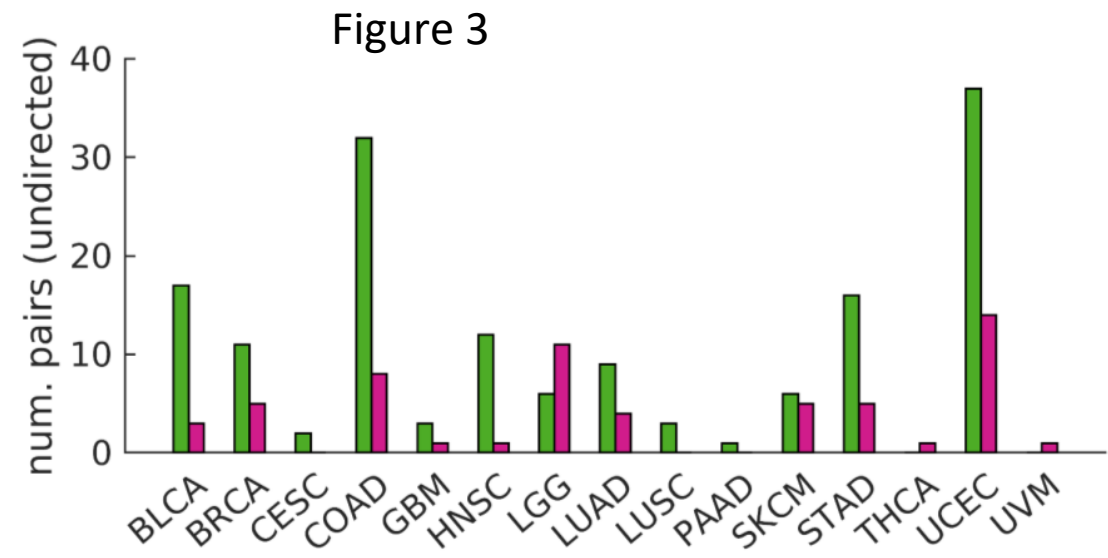

b

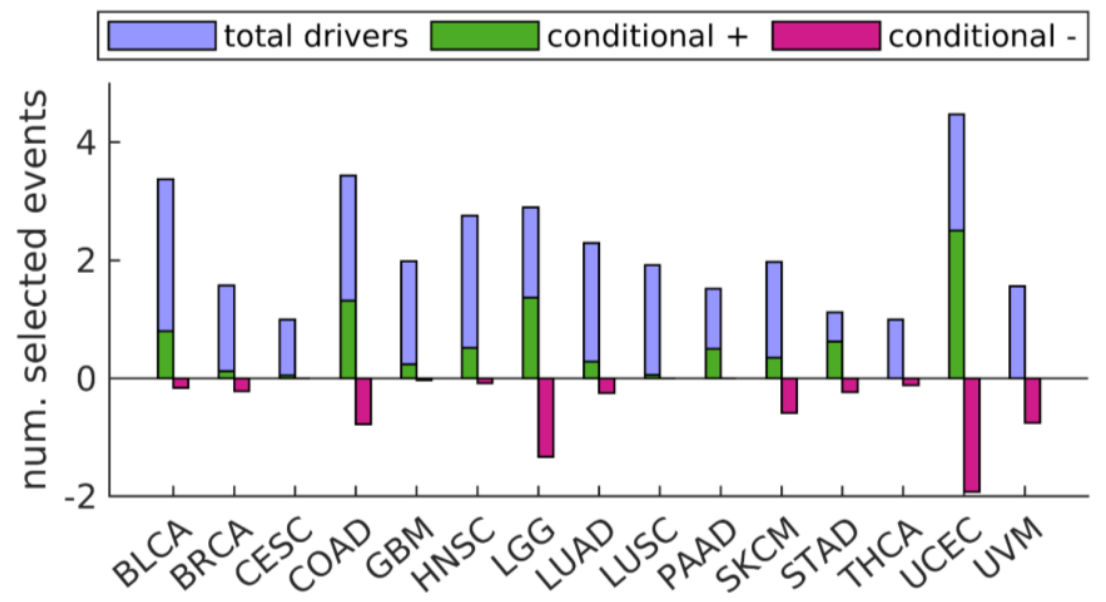

C

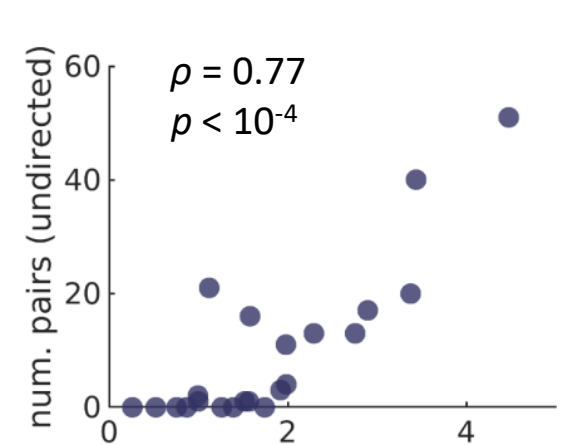

num. driver substitutions d

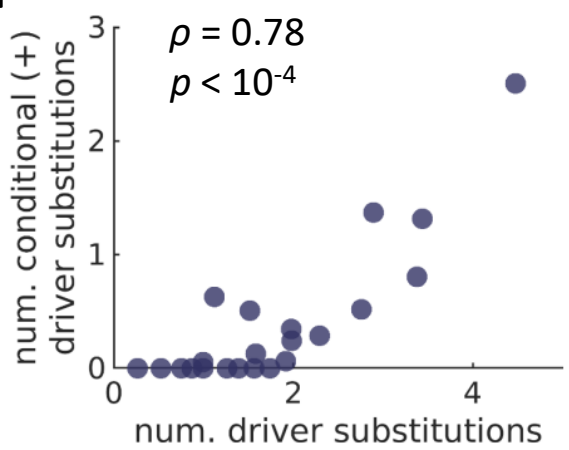

e

gene pairs found in $>1$ cancer type

\begin{tabular}{ll}
\hline ARID1A - ERBB3 & BLCA, STAD \\
ATRX - IDH1 & GBM, LGG \\
IDH1 - TP53 & GBM, LGG \\
PGM5 - RNF43 & COAD, STAD \\
RB1 - TP53 & BLCA, GBM \\
ARID1A - TP53 & STAD, UCEC \\
BRAF - KRAS & COAD, LUAD \\
BRAF - NRAS & SKCM, THCA \\
IDH1 - PTEN & GBM, LGG \\
KRAS - TP53 & LUAD, UCEC \\
PIK3CA - TP53 & BRCA, COAD, STAD
\end{tabular}

f

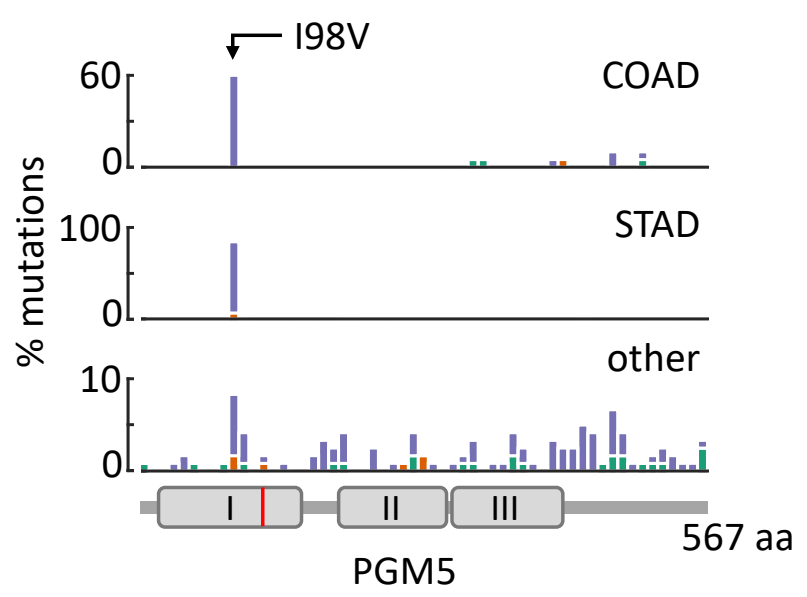




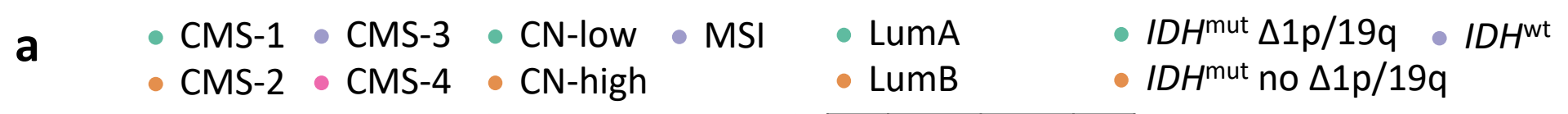
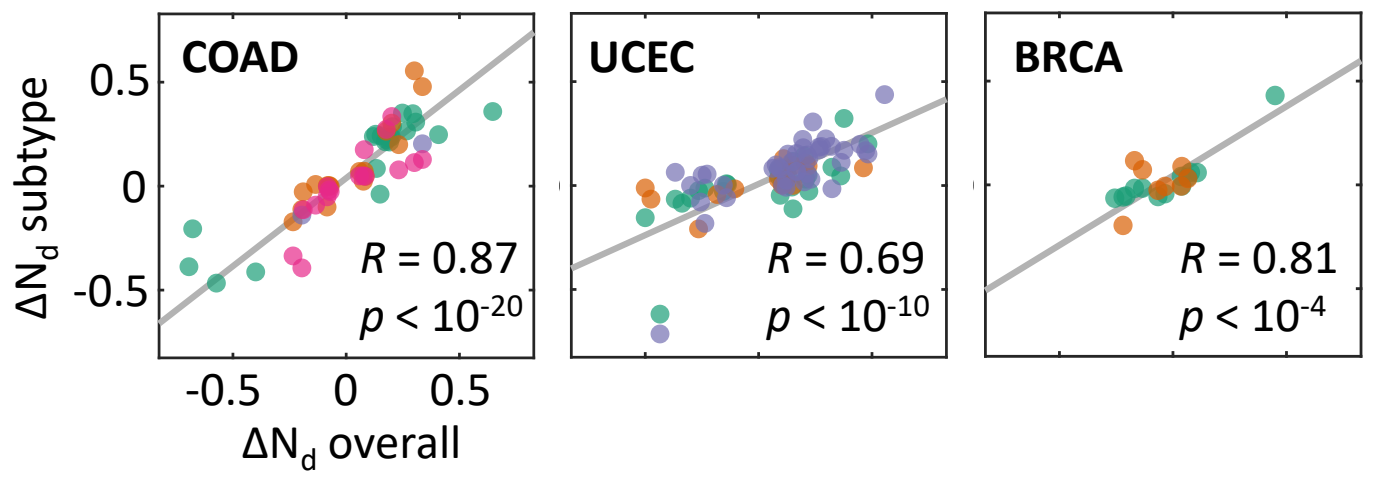

b gene $A-$ gene $C$
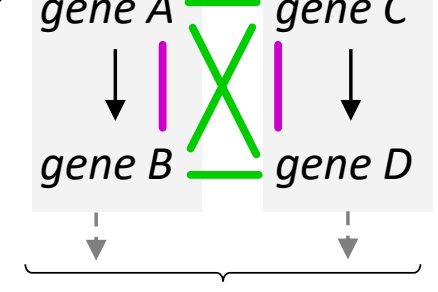

cancer progression

C gene $A \ldots \ldots$ cancer

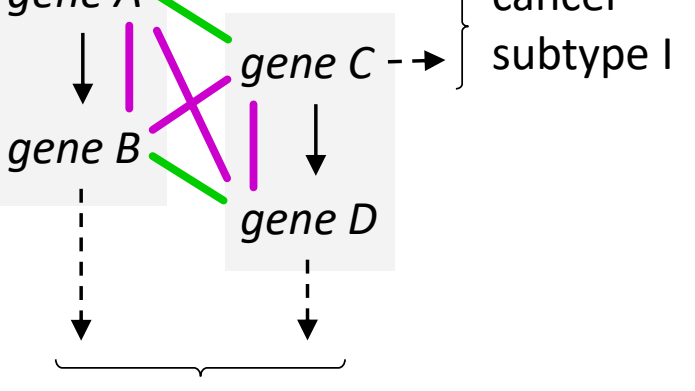

cancer subtype II

Figure 5

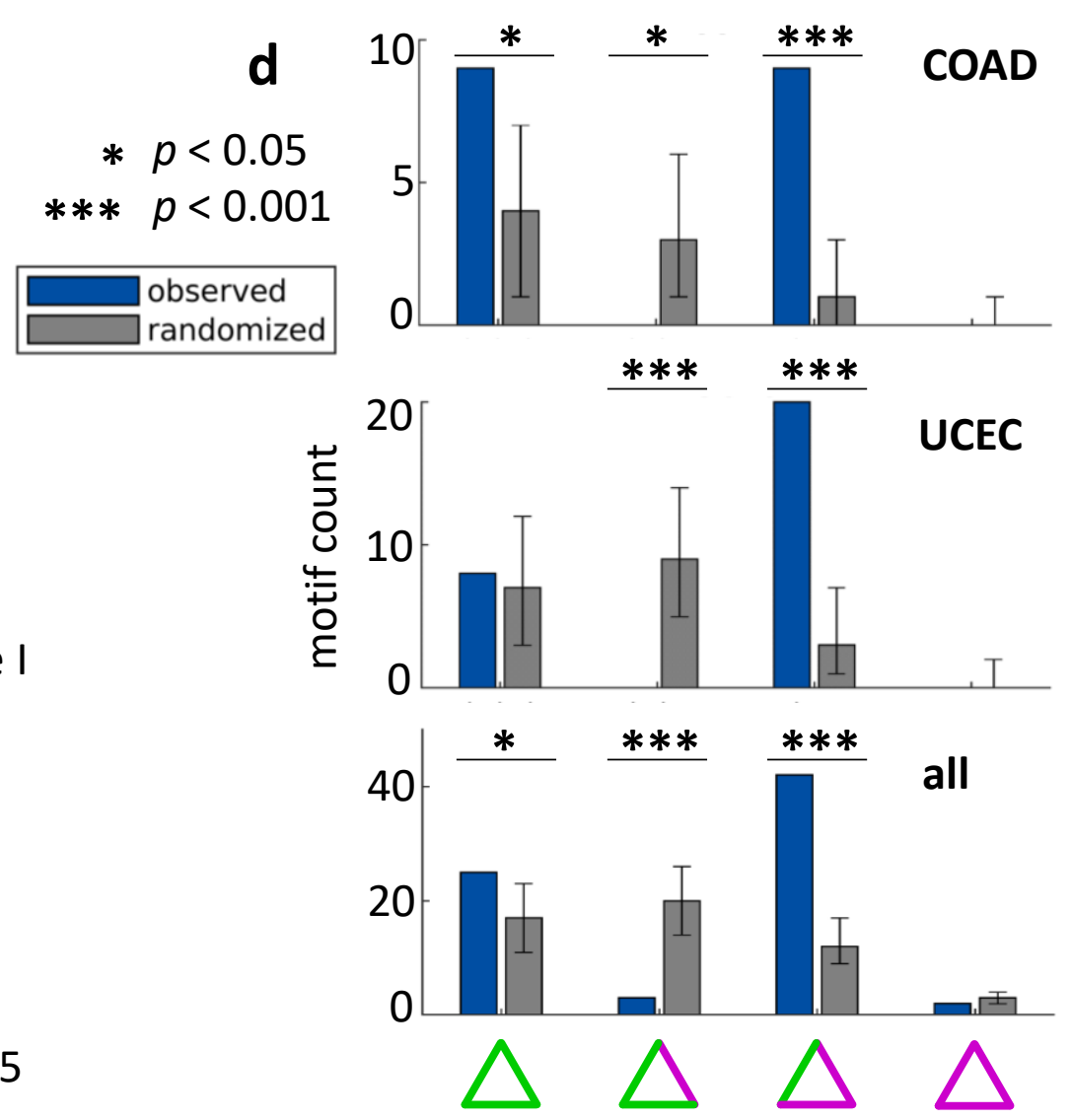




\section{epistasis} network
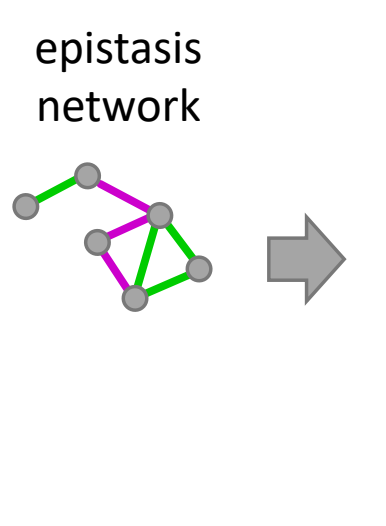

$$
\text { (0. }
$$

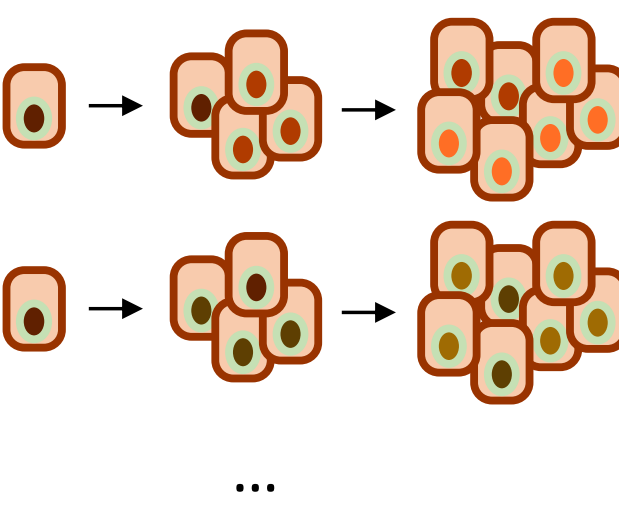

$$
\begin{aligned}
& \nabla \infty<0
\end{aligned}
$$

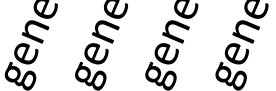

b

epistasis network

mutation-based subtypes

Figure 6

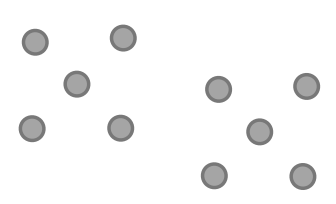

บิ $14 \%$

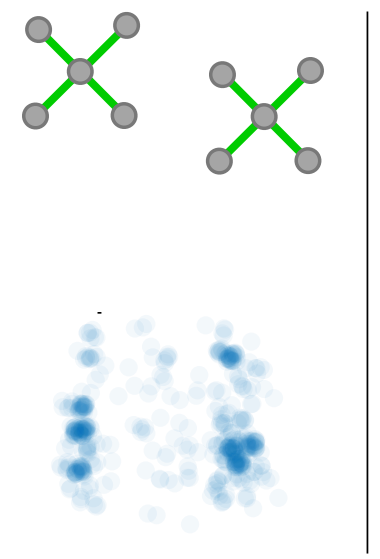

$27 \%$

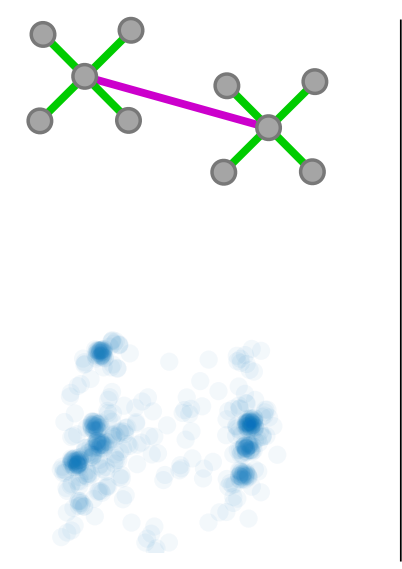

$28 \%$

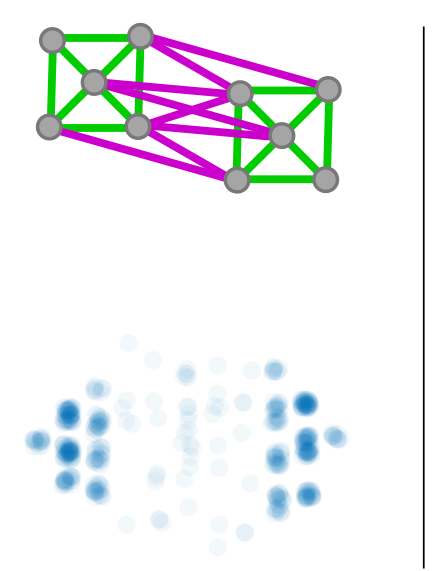

$54 \%$

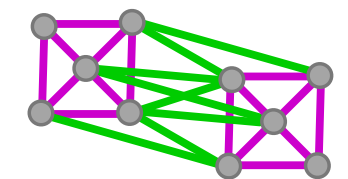

$11 \%$

$\%$ mutational variance explained by PC1 


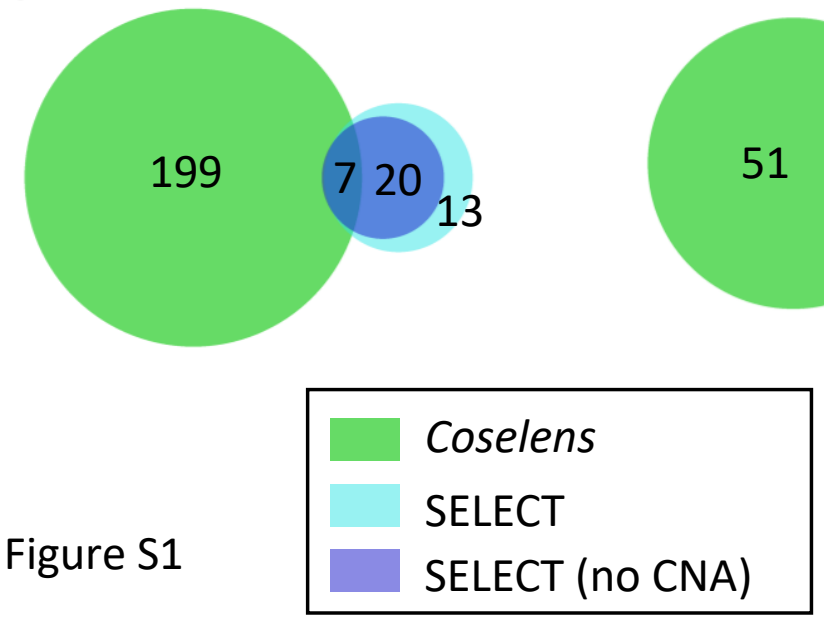




\section{stomach}

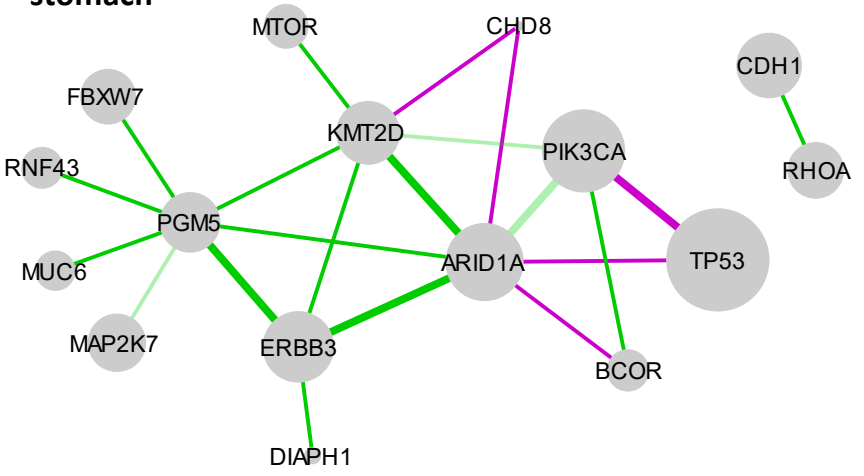

cervix

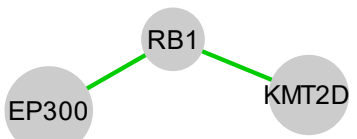

thyroid

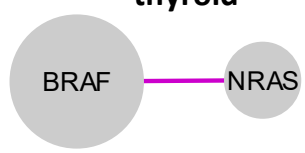

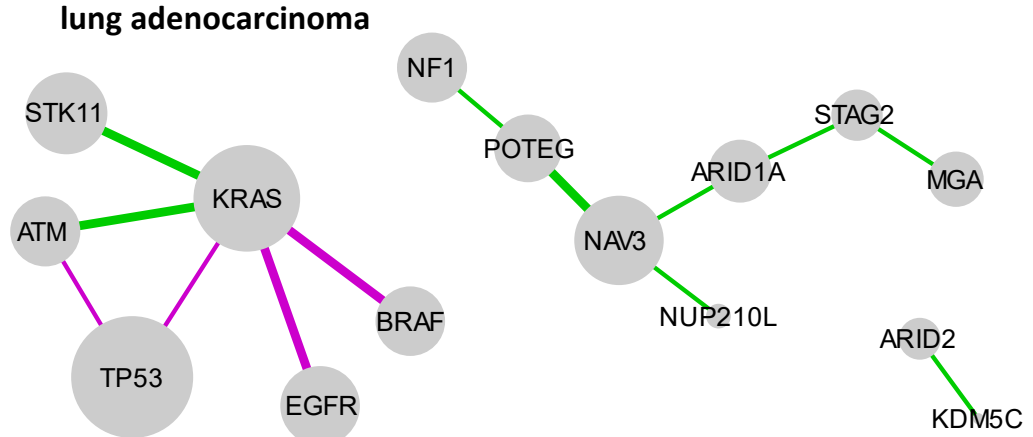

head - neck squamous
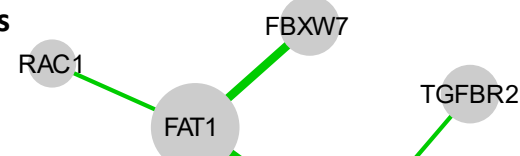

conkenantive

CDKN2A.p14arf
TP53

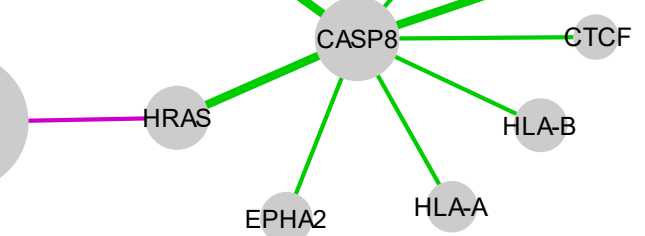

skin cutaneous

melanoma
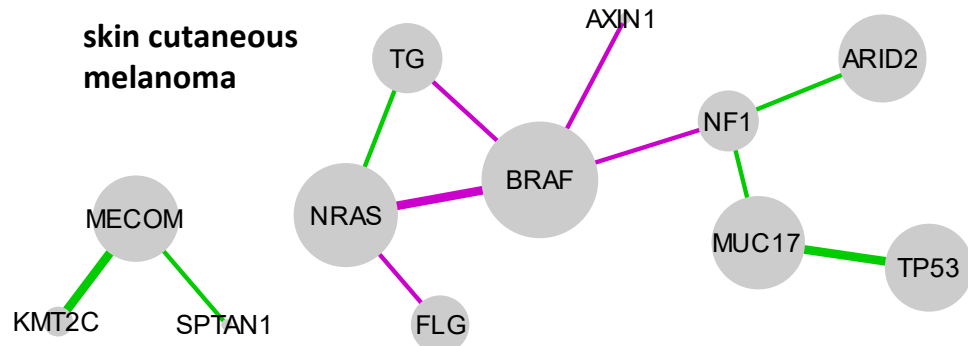

breast

TBX3
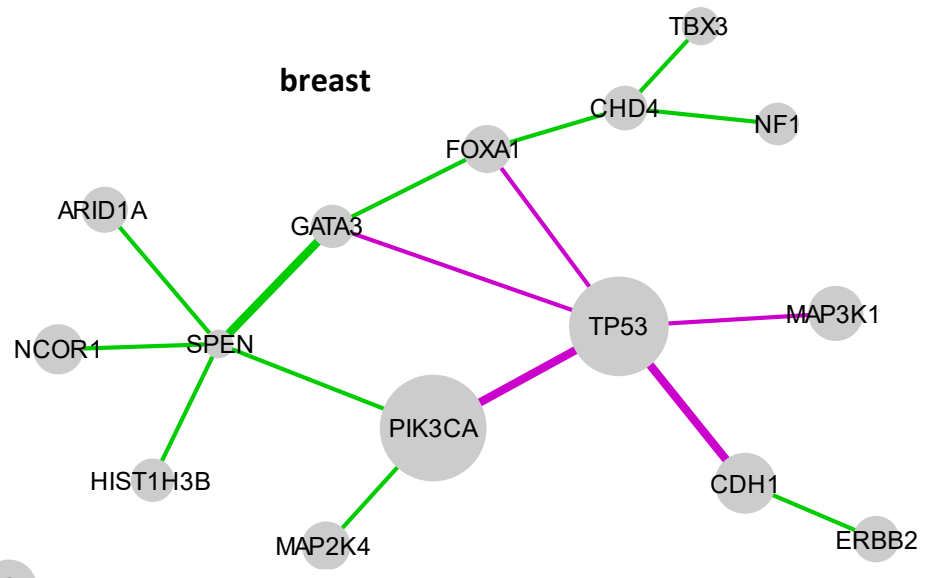

pancreas

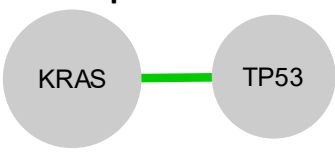

uveal melanoma

GNAQ GNA11

lung squamous
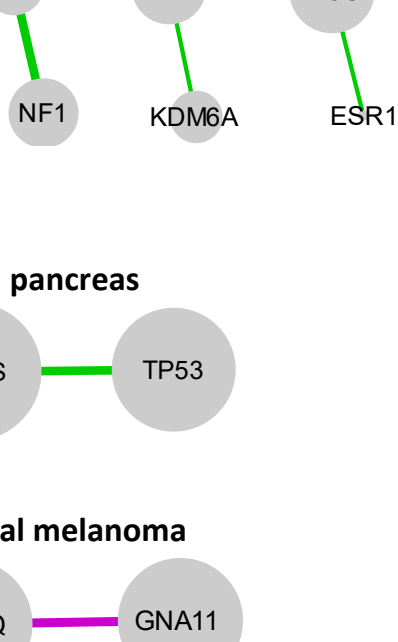

RASA1 CDH10 PIK3CA

\section{CDKN2A.p16INK4a}

bladder

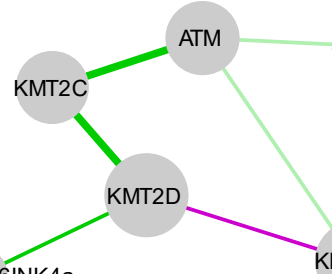

KDM6A
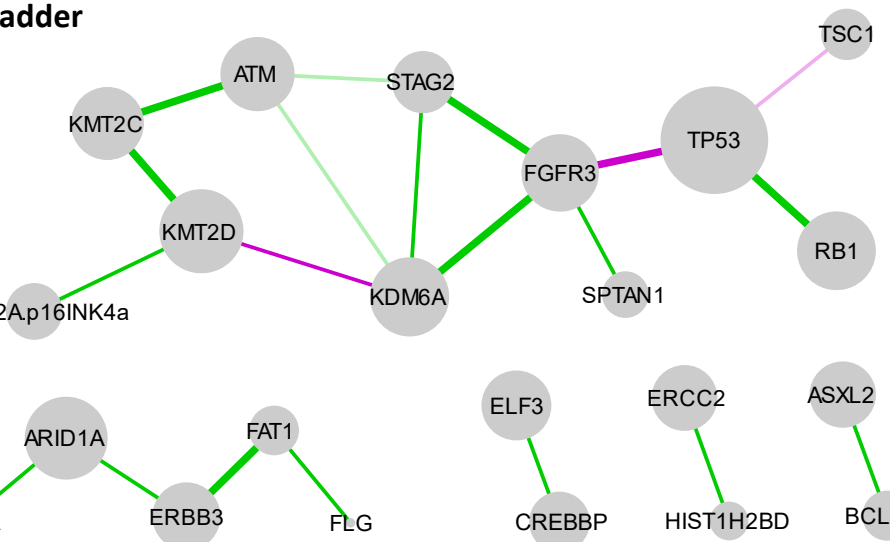

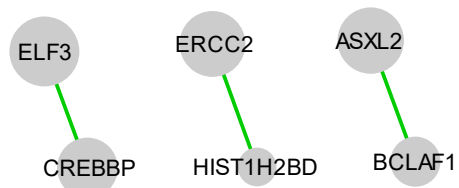




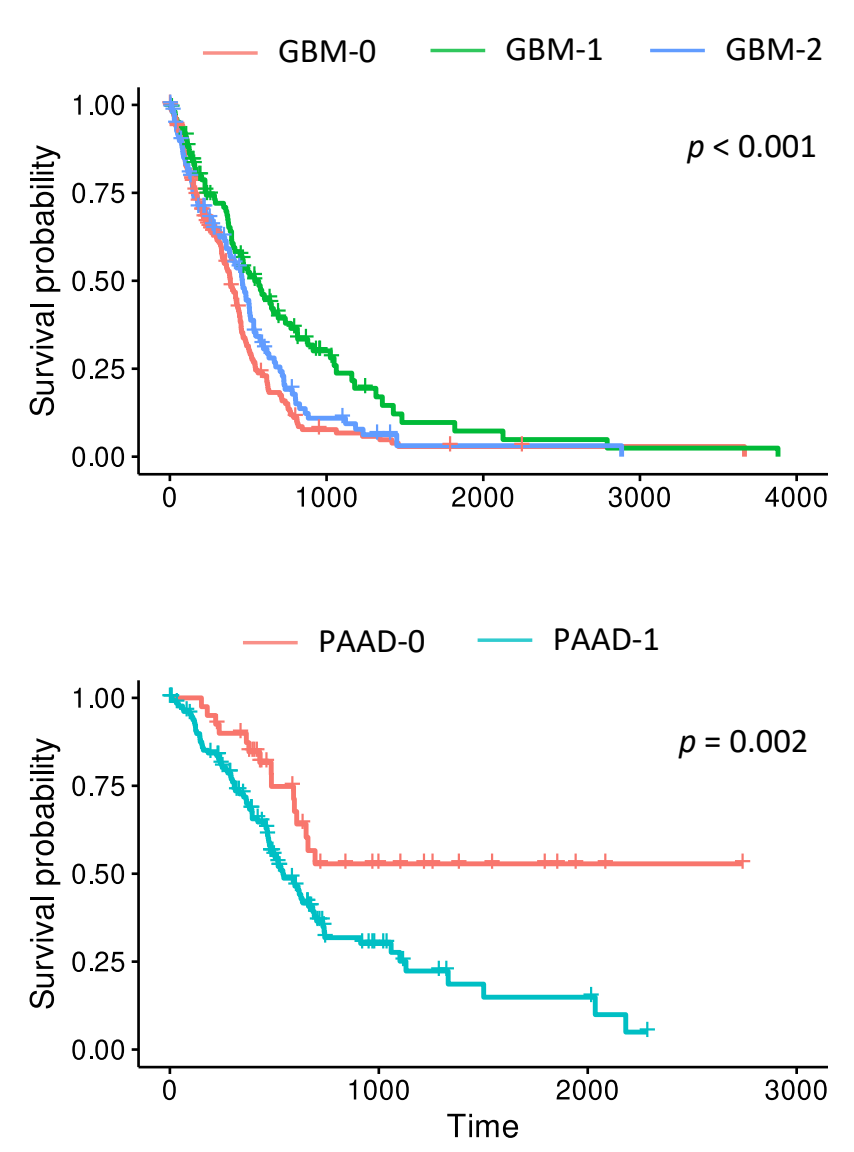

$=$ LGG-0 $=$ LGG-1 $=$ LGG-2

- LGG-3 — LGG-4 — LGG-5

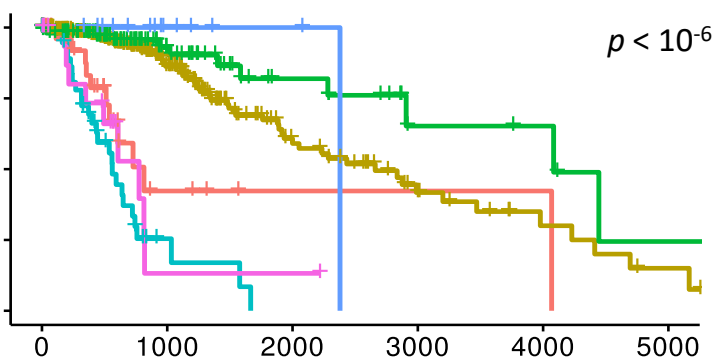

Figure S3

UCEC-1 — UCEC-5 — UCEC-6

- HNSC-0 HNSC-1 HNSC-2
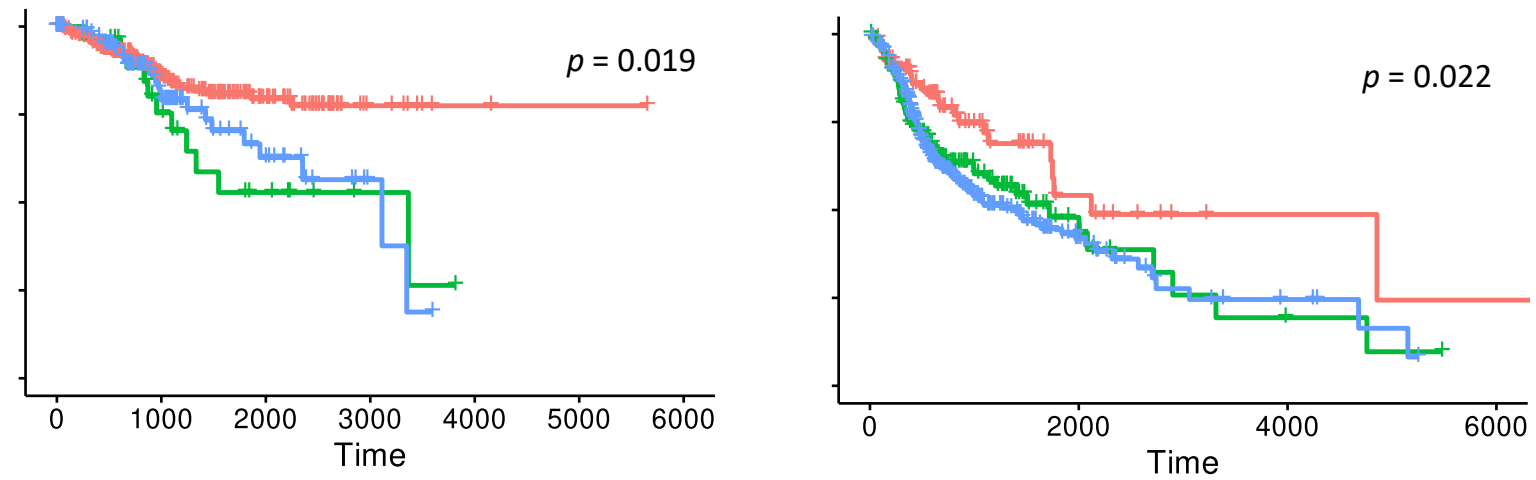


\section{Figure S4}
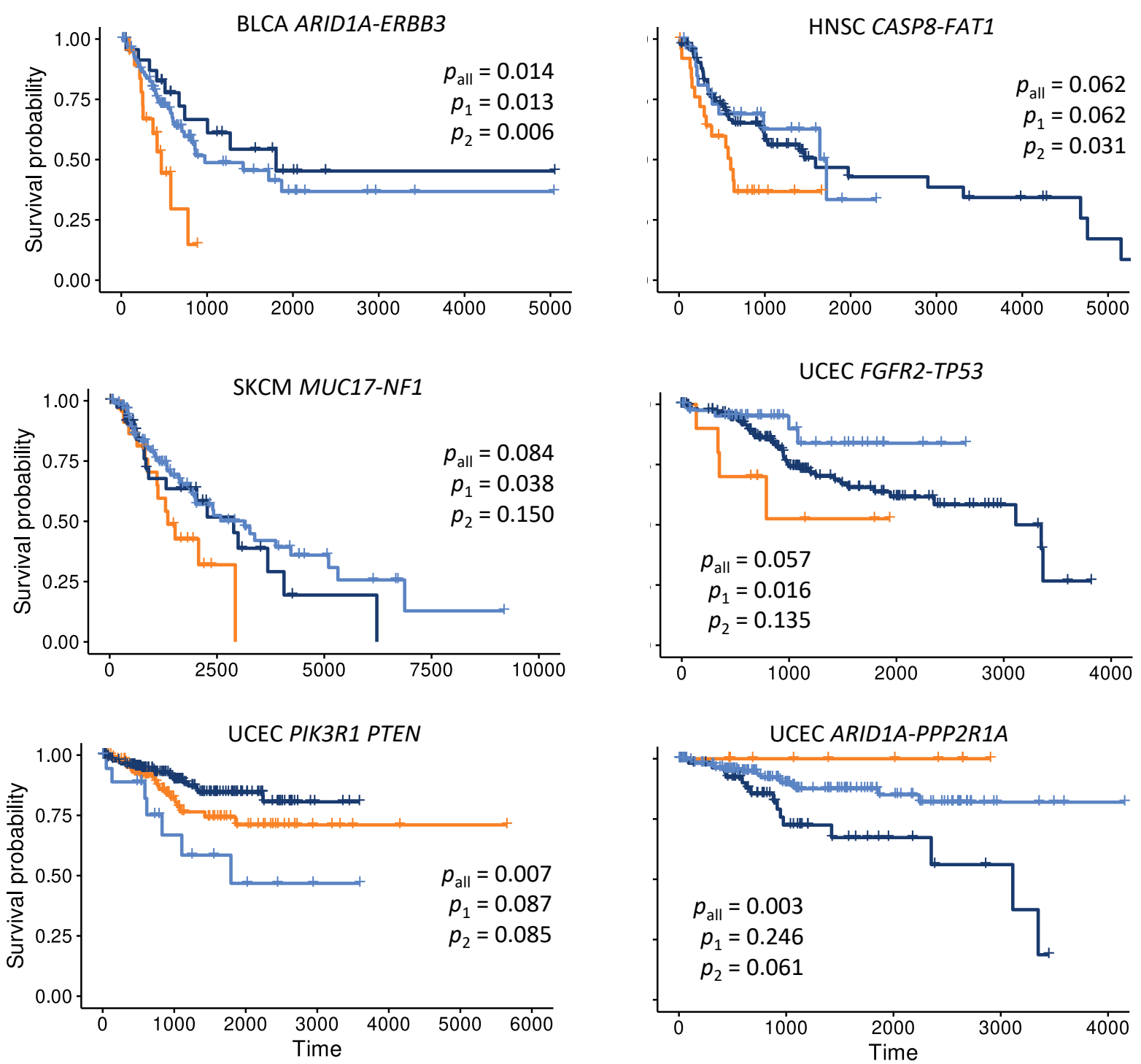

LGG IDH1-TP53

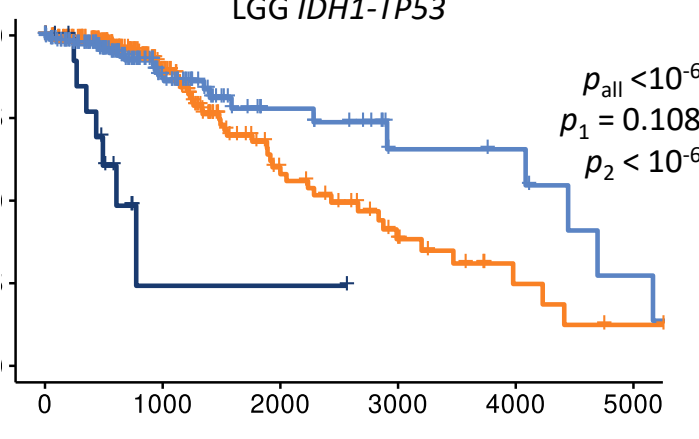

UCEC ARID1A-PTEN
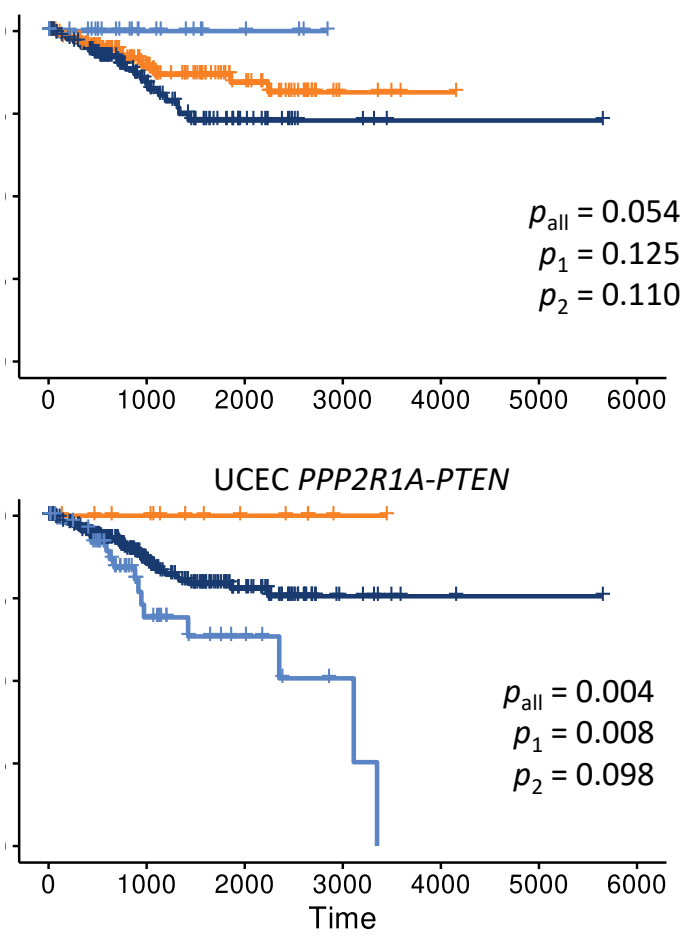